\title{
Observables in the Guarino-Jafferis-Varela/CS-SYM duality
}

\author{
Thiago R. Araujo ${ }^{a, b}$ and Horatiu Nastase ${ }^{a}$ \\ ${ }^{a}$ Instituto de Física Teórica, UNESP-Universidade Estadual Paulista, \\ R. Dr. Bento T. Ferraz 271, Bl. II, Sao Paulo 01140-070, SP, Brazil \\ ${ }^{b}$ Asia Pacific Center for Theoretical Physics, POSTECH, \\ Cheongam-ro Nam-gu 37673, Pohang-si, Korea \\ E-mail: thiago.araujo@apctp.org, nastase@ift.unesp.br
}

ABSTRACT: We study various semiclassical observables in the duality proposed by Guarino, Jafferis and Varela, between a warped $A d S_{4} \times$ squashed $S^{6}$ gravitational solution and a 3 dimensional $\mathcal{N}=2 \mathrm{SYM}-\mathrm{CS}_{k}$ conformal gauge theory, deformed from the maximal $\mathrm{SU}(N)$ $\mathcal{N}=8$ SYM. Baryon vertices corresponding to particle-like branes have unusual behaviour with $N$ and $k$ and present strong evidence for a certain level-rank duality. Wilson loops and the anomalous dimensions of operators of high spin scale like $(N / k)^{3 / 2}$. The entanglement entropy behaves like in a usual CFT. Giant magnon operators obey the same law as in 4 dimensional $\mathcal{N}=4 \mathrm{SYM}$, and giant gravitons are also sub-determinant operators.

KEYwords: AdS-CFT Correspondence, D-branes, Long strings

ARXIV EPRINT: 1609.08008 


\section{Contents}

1 Introduction $\quad 2$

2 Guarino-Jafferis-Varela solution and duality to SYM-CS 3

2.1 Quantization of charges 5

$\begin{array}{lll}2.2 & \text { Field theory dual } & 8\end{array}$

3 Particle-like branes and their field theory duals $\quad 9$

3.1 Branes on cycles in the GJV geometry 9

$\begin{array}{lll}3.2 & \text { Field theory dual: baryon operators } & 14\end{array}$

$\begin{array}{lll}3.3 & \text { Brane stability and operator bounds } & 15\end{array}$

$\begin{array}{ll}3.4 & \text { Reducing the number of fundamental strings } \\ \end{array}$

$\begin{array}{ll}3.5 & \text { Giant gravitons: gravity calculation } \\ \end{array}$

3.5.1 D2-brane on $S^{2} \subset A d S_{4} \quad 20$

3.5.2 D2-brane on the squashed $S^{2} \subset \mathcal{S} S^{5} \quad 21$

3.5.3 D4-brane on the squashed $\mathbb{C P}^{2} \subset \mathcal{S} S^{5} \quad 23$

3.6 Giant gravitons: field theory operators 24

3.7 Gauge coupling from wrapped brane 24

4 Strings in the geometry $\quad \mathbf{2 5}$

4.1 Wilson loops 25

$\begin{array}{lll}4.2 & \text { Operators of large spin } & 26\end{array}$

5 Entanglement entropy $\quad 26$

6 Giant magnons $\quad 28$

6.1 Gravity calculation 28

6.1.1 Review of $A d S_{5} \times S^{5}$ giant magnons 28

$\begin{array}{ll}\text { 6.1.2 Giant magnons in the gravity dual } & 29\end{array}$

$\begin{array}{lll}6.2 & \text { Field theory } 30\end{array}$

6.2.1 Hamiltonian and diagonalization 30

$\begin{array}{lll}7 & \text { Conclusions } & 32\end{array}$

$\begin{array}{ll}\text { A Conventions } & 33\end{array}$ 


\section{Introduction}

The AdS/CFT correspondence has proved very successful in obtaining the nonperturbative properties of strongly coupled field theories. The best understood is the one originally studied by Maldacena, the duality of 4 dimensional $\mathrm{SU}(N) \mathcal{N}=4$ SYM vs. string theory in $A d S_{5} \times S^{5}$, which has been used as a toy model for the physics of strong interactions, i.e. QCD [1]. On the other hand, in 3 dimensions, the best understood duality is of the ABJM model vs. string theory in $A d S_{4} \times \mathbb{C P}^{3}$ background [2], which has been used as a toy model for 3 dimensional condensed matter systems. The ABJM model is an $\mathcal{N}=6$ superconformal theory with gauge group $\mathrm{SU}(N) \times \mathrm{SU}(N)$ and Chern-Simons gauge fields, which is why it is more useful for condensed matter than for particle physics. In both SYM and ABJM cases a very large number of different types of calculations have been performed, due to the simplicity of the theories, yet in both the theories are very special (they are toy models), so the applicability of the lessons learned is unclear.

Recently however, a very interesting duality has been proposed in 3 dimensions by Guarino, Jafferis and Varela, between a fixed point of an $\mathcal{N}=2$ supersymmetric $\operatorname{SU}(N)$ SYM gauge theory with a Chern-Simons term of level $k$ added and string theory in a certain warped, squashed $A d S_{4} \times S^{6}$ background of massive type IIA theory [3]. The theory is still superconformal, but has less supersymmetry, has an extra parameter $k$ that forms a 't Hooft coupling $\lambda=N / k$, and has both the SYM term interesting for particle physics and a CS term at level $k$ that as we will see can dominate the IR and imply a certain interesting level-rank duality. The theory arises as a deformation of the maximal $\mathcal{N}=8 \mathrm{SU}(N) \mathrm{SYM}$ (with adjoint fields) on the worldvolume of $N$ D2-branes (see also [4]), and the resulting SCFT has been studied in [5].

The logic that led to the GJV solution was as follows. The four dimensional $\mathcal{N}=8$ $\mathrm{SO}(8)$-gauged supergravity [6] arises as a consistent truncation of 11 dimensional supergravity (low energy of M-theory) on the seven sphere [7, 8]. But it was pointed out in [9-11] that the original de Wit-Nicolai solution [6] is, in fact, just one particular point in a continuous one-parameter family of solutions, where the parameter $\omega \in[0, \pi / 8]$ determines the linear combination of the $\mathrm{SO}(8)$ electric and magnetic gauge fields. In this framework, the de Wit-Nicolai solution is purely electric and can be embedded into the $D=11$ supergravity as a consistent truncation. These dyonic gaugings cannot be embedded in string/M-theory, as recently proved in [12]. A related symmetry group, namely the $\operatorname{ISO}(7)=\mathrm{SO}(7) \ltimes \mathbb{R}^{7}$, can be obtained from $\mathrm{SO}(8)$ through a Inönü-Wigner contraction [13]. Solutions with this symmetry also admit dyonic gaugings [10], studied further in [14], and it has been proved [15] that the purely electric solutions can be embedded into $D=11$ supergravity as a consistent truncation on $S^{6} \times S^{1}$. Moreover, the dyonic ISO(7) gauged SUGRA solutions arise as consistent truncations of massive type IIA solution on a squashed 6-sphere (shown also in [16]), that we denote by $\mathcal{S} S^{5}$, and preserve up to $\mathcal{N}=3$ supersymmetries $[3,17-19]$ of the total $\mathcal{N}=8$. The magnetic coupling constant $m$ is identified with the Romans mass $F_{0}$. In fact, this solution has fixed points that uplift to $\mathcal{N}=2 A d S_{4} \times \mathcal{S} S^{5}$. Since the internal space has the topology of a sphere, we expect that this solution arises as the near 
horizon geometry of a stack of $N$ D2-branes, with the Romans mass inducing a Chern Simons term on the brane worldvolume. ${ }^{1}$

In this paper, we will study the GJV duality from the point of view of various observables associated with semiclassical string theory objects in the gravity dual. Static D-branes wrapped on cycles in the geometry correspond, if there are nontrivial tadpoles, to baryon vertices in the field theory, the (external) quarks corresponding to long strings that must end on the D-brane. Moving D-branes wrapped on cycles correspond to giant gravitons (gravitons that polarize into nontrivial D-branes in the geometry), and correspond to certain subdeterminant operators. Long strings in the geometry can give Wilson loops, the anomalous dimension of high spin operators and giant magnon spin chain operators.

The paper is organized as follows. In section two we review the GJV solution, perform the quantization of the charges, and review the dual field theory. In section 3 we study D-branes on cycles and their gravity duals: baryon operators for the static branes and determinant operators for moving branes, i.e. giant gravitons, as well as the gauge coupling on the wrapped branes. In section 4 we study entanglement entropy from the holographic prescription. In section 5 we study strings in the geometry, giving Wilson loops and the anomalous dimension of operators of large spin. In section 6 we study giant magnons from the point of view of both gravity and field theory, and in section 7 we conclude.

\section{Guarino-Jafferis-Varela solution and duality to SYM-CS}

In [3] the authors showed that a consistent truncation of a massive type IIA SUGRA on the six sphere $S^{6}$ gives rise to the ISO(7)-dyonically-gauged SUGRA with the magnetic coupling constant related to the Romans mass. They also showed that this supergravity solution has a fixed point which uplifts to a new $\mathcal{N}=2 A d S_{4} \times \mathcal{S} S^{5}$ solution, where $\mathcal{S} S^{5}$ is the suspension of $S^{5}$ and is topologically equivalent to the six sphere $S^{6}$ [20].

The fixed point solution $[3,20]$ has metric (in the Einstein frame and the conventions of $[21])$

$$
\mathrm{d} s_{\mathrm{IIA}}^{2}=\Delta\left\{\mathrm{d} s_{A d S_{4}}^{2}+\frac{3}{2} \mathrm{~d} \alpha^{2}+\Xi \mathrm{d} s_{\mathbb{C P}^{2}}^{2}+\Omega \eta^{2}\right\}
$$

where $\Delta=L^{2}(3+\cos 2 \alpha)^{1 / 2}(5+\cos 2 \alpha)^{1 / 8}, \Xi=6 \sin ^{2} \alpha /(3+\cos 2 \alpha), \Omega=9 \sin ^{2} \alpha /(5+$ $\cos 2 \alpha)$ and $\alpha \in[0, \pi]$. Also $\eta=\mathrm{d} \psi+\omega$, where $\psi \in[0,2 \pi]$ and $\mathcal{J}=\frac{1}{2} \mathrm{~d} \omega$ is the Kähler-form of $\mathbb{C P}^{2}$, which is parameterized by the coordinates $(\lambda, \theta, \phi, \sigma)$, we refer to appendix $\mathrm{A}$ for further details. Finally, we write the warp factor in string frame as

$$
L_{\text {string }}^{2}=e^{\phi / 2} \Delta=L^{2} e^{\phi_{0} / 2}(5+\cos 2 \alpha)^{1 / 2} .
$$

The determinant of the internal manifold metric

$$
\mathrm{d} s_{\mathcal{S} S^{5}}^{2}=\frac{3}{2} \mathrm{~d} \alpha^{2}+\Xi \mathrm{d} s_{\mathbb{C P}^{2}}^{2}+\Omega \eta^{2}
$$

\footnotetext{
${ }^{1}$ A related duality conjecture has been considered in [20], where the authors studied a stack of $D 2$ branes probing a Calabi-Yau threefold singularity in the massive type IIA supergravity. In this case, the field theory is an $\mathcal{N}=2$ Chern-Simons quiver gauge theory $\mathrm{U}(N)^{G}$, where $G$ is the Euler number of the resolved manifold. The authors in [20] argued that in the low energy, the D2-brane theory flows to a modification (resolution) of the gravity dual $\mathcal{N}=2 A d S_{4} \times \mathcal{S} S^{5}$ of GJV [3].
} 
is

$$
\operatorname{det}\left(G_{\mathcal{S} S^{5}}\right)=\frac{3 \Xi^{4} \Omega}{128} \sin ^{2} \theta \sin ^{6} \lambda \cos ^{2} \lambda
$$

For fixed $\psi$ and $\alpha$ (with $\alpha \neq 0, \pi$ to avoid the isolated conical singularities), the internal manifold has a $\mathbb{C P}^{2}$ space, and for $\lambda=\pi / 2$ and fixed $\sigma$ it has a $\mathbb{C P}^{1}$.

The solution also contains the fields

$$
\begin{aligned}
e^{\phi} & =e^{\phi_{0}} \frac{(5+\cos 2 \alpha)^{3 / 4}}{(3+\cos 2 \alpha)} \\
H_{3} & =24 \sqrt{2} L^{2} e^{\phi_{0} / 2} \frac{\sin ^{3} \alpha}{(3+\cos 2 \alpha)^{2}} \mathcal{J} \wedge \mathrm{d} \alpha
\end{aligned}
$$

in the NS-NS sector. For later convenience, we write $e^{\phi} \equiv g_{s}^{\alpha}, e^{\phi_{0}} \equiv g_{s}$ and $\gamma(\alpha) \equiv$ $\frac{(5+\cos 2 \alpha)^{3 / 4}}{(3+\cos 2 \alpha)}$. With this notation, equation $(2.1 \mathrm{e})$ becomes

$$
g_{s}^{\alpha}=g_{s} \gamma(\alpha)
$$

We note that the string coupling $g_{s}^{\alpha}$ is maximum at $\alpha=\pi / 2$ and minimum at $\alpha=0, \pi$ (its derivative with respect to $\alpha$ is positive on the interval $[0, \pi / 2]$ and negative on the interval $[\pi / 2, \pi])$.

The RR fields are ${ }^{2}$

$$
\begin{gathered}
\widetilde{F}_{0}=3^{-1 / 2} L^{-1} e^{-5 \phi_{0} / 4} \equiv m \\
\widetilde{F}_{2}=-\sqrt{6} L e^{-3 \phi_{0} / 4}\left\{\frac{4 \sin ^{2} \alpha \cos \alpha}{(3+\cos 2 \alpha)(5+\cos 2 \alpha)} \mathcal{J}+\frac{3(3-\cos 2 \alpha)}{(5+\cos 2 \alpha)^{2}} \sin \alpha \mathrm{d} \alpha \wedge \eta\right\} \\
=-\sqrt{6} L e^{-3 \phi_{0} / 4}\left\{\frac{4 \sin ^{2} \alpha \cos \alpha}{(3+\cos 2 \alpha)(5+\cos 2 \alpha)} \mathcal{J}-\mathrm{d}\left(\frac{3 \cos \alpha}{5+\cos 2 \alpha}\right) \wedge \eta\right\} \\
\widetilde{F}_{4}=L^{3} e^{-\phi_{0} / 4}\left\{6 \operatorname{vol}\left(A d S_{4}\right)-12 \sqrt{3} \frac{(7+3 \cos 2 \alpha)}{(3+\cos 2 \alpha)^{2}} \sin ^{4} \alpha \operatorname{vol}\left(\mathbb{C P}^{2}\right)\right. \\
\left.+18 \sqrt{3} \frac{(9+\cos 2 \alpha) \sin ^{3} \alpha \cos \alpha}{(3+\cos 2 \alpha)(5+\cos 2 \alpha)} \mathcal{J} \wedge \mathrm{d} \alpha \wedge \eta\right\}
\end{gathered}
$$

in the R-R sector. Finally, the constants are

$$
L^{2} \equiv 2^{-5 / 8} 3^{-1} g^{-25 / 12} m^{1 / 12} \quad \text { and } \quad e^{\phi_{0}} \equiv 2^{1 / 4} g^{5 / 6} m^{-5 / 6}
$$

\footnotetext{
${ }^{2}$ The conventions of [3] imply the relation $2 \operatorname{vol}\left(\mathbb{C P}^{2}\right)=\mathcal{J}^{\text {(theirs) }} \wedge \mathcal{J}^{\text {(theirs) }}$ while our conventions imply $2 \operatorname{vol}\left(\mathbb{C P}^{2}\right)=i^{2} \mathcal{J}^{\text {(ours) }} \wedge \mathcal{J}^{\text {(ours) }}$. Therefore, the 4-form potential has a difference of sign compared to the original solution. We thank Georgios Itsios and the anonymous referee for pointing out this difference of conventions.
} 
Using the definitions of [22], where $H_{3}=\mathrm{d} B, \widetilde{F}_{2}=\mathrm{d} C_{1}+\widetilde{F}_{0} B$ and $\widetilde{F}_{4}=\mathrm{d} C_{3}+C_{1} \wedge$ $H_{3}+\frac{\widetilde{F}_{0}}{2} B \wedge B$, the potentials that generate these $p$-forms are given by [17]

$$
\begin{aligned}
B & =-\frac{6 L^{2} e^{\phi_{0} / 2} \sqrt{2} \sin ^{2} \alpha \cos \alpha}{(3+\cos 2 \alpha)} \mathcal{J}-\frac{3 L^{2} e^{\phi_{0} / 2}}{\sqrt{2}} \sin \alpha \mathrm{d} \alpha \wedge \eta \\
C_{1} & =\frac{L e^{-3 \phi_{0} / 4} \sqrt{6} \sin ^{2} \alpha \cos \alpha}{(5+\cos 2 \alpha)} \eta \equiv 2 a_{0}(\alpha) \eta \\
C_{3} & =L^{3} e^{-\phi_{0} / 4}\left(6 \Gamma+\frac{6 \sqrt{3} \sin ^{4} \alpha}{(3+\cos 2 \alpha)} \mathcal{J} \wedge \eta\right),
\end{aligned}
$$

where $\mathrm{d} \Gamma=\operatorname{vol}\left(A d S_{4}\right)$, then $\Gamma=-\frac{1}{3} r^{3} \sin \vartheta \mathrm{d} t \wedge \mathrm{d} \vartheta \wedge \mathrm{d} \varphi$. Furthermore, the field strengths satisfy the Bianchi identity

$$
\begin{aligned}
\mathrm{d} H_{3} & =0 \\
\mathrm{~d} F_{2} & =\widetilde{F}_{0} H_{3} \\
\mathrm{~d} F_{4} & =H_{3} \wedge \widetilde{F}_{2} .
\end{aligned}
$$

More generally, we define the gauge-invariant field strength $\widetilde{F}_{p}$ as

$$
\widetilde{F}_{p}=\left(\mathrm{d} C-H_{3} \wedge C+F_{0} e^{B}\right)_{p},
$$

and we see that the $p$-forms in the RR-sector are not closed, but satisfy the modified Bianchi identity $\mathrm{d} \widetilde{F}_{k}=H_{3} \wedge \widetilde{F}_{k-2}$, that is, the $p$-forms are closed under the derivative $\mathrm{d}_{H}=\mathrm{d}-H_{3} \wedge$.

\subsection{Quantization of charges}

We can consider the most appropriate flux in a supergravity background by the so called, Page charges [23], defined as follows. Consider the $d$-closed $p$-form defined as

$$
\widehat{F}_{p}:=\left[e^{-B} \wedge\left(\widetilde{F}_{0}+\widetilde{F}_{2}+\widetilde{F}_{4}+\widetilde{F}_{6}+\widetilde{F}_{8}\right)\right]_{p} .
$$

Then the Page charges $N_{k} \in \mathbb{Z}$ are defined by

$$
n_{p}:=\frac{1}{\left(2 \pi \ell_{s}\right)^{p-1}} \int_{\Sigma^{p}} \widehat{F}_{p}
$$

where $\Sigma^{p}$ is a closed space-like $p$-cycle and $l_{s}=\sqrt{\alpha^{\prime}}$. In particular,

$$
k \equiv n_{0}=2 \pi \ell_{s} F_{0}=2 \pi \ell_{s} m .
$$

is an integer that has a simple interpretation in the field theory as a CS level. In the solution considered in the last section, we have, on a 6-cycle (the whole compact space)

$$
N \equiv n_{6}=\frac{1}{\left(2 \pi \ell_{s}\right)^{5}} \int_{\mathcal{S} S^{5}}\left(\widetilde{F}_{(6)}-B \wedge \mathrm{d} C_{(3)}-\frac{\widetilde{F}_{(0)}}{6} B \wedge B \wedge B\right),
$$


where $\widetilde{F}_{(6)}=-e^{\phi / 2} * \widetilde{F}_{4}$, and the Hodge dual is taken with relation to the Einstein frame metric (2.1a), as the authors of [3] have shown. This integer will correspond to the rank of the gauge group in the field theory. In fact, these are the quantized charges that we have associated to the massive supergravity above, and the quantization of the fluxes implies that

$$
\begin{aligned}
L & =\frac{\pi^{3 / 8} \ell_{s}}{2^{7 / 48} 3^{7 / 24}}\left(k N^{5}\right)^{1 / 24} ; \quad e^{\phi_{0}}=\frac{2^{11 / 12} \pi^{1 / 2}}{3^{1 / 6}} \frac{1}{\left(k^{5} N\right)^{1 / 6}} \Rightarrow \\
L_{\text {string }}^{2} & =\frac{2^{1 / 6} \pi}{3^{2 / 3}}\left(\frac{N}{k}\right)^{1 / 3} \ell_{s}^{2} \sqrt{5+\cos 2 \alpha} .
\end{aligned}
$$

as the authors $[17,20]$ have shown.

Note that

$$
L_{\text {string }} e^{\phi_{0}}=\frac{2 \pi \ell_{s}}{\sqrt{3} k}[5+\cos 2 \alpha]^{1 / 4} \Rightarrow L_{\text {string }} g_{s}^{\alpha}=\frac{2 \pi \ell_{s}}{k} \frac{5+\cos 2 \alpha}{\sqrt{3}(3+\cos 2 \alpha)} .
$$

In [23], the author showed that there are three distinct notions of charges associated to a given gauge field, namely, Brane charges, Maxwell charges and Page charges, and only the latter is quantized, as we have seen above.

Consider, for example, the 4-form field strength $\widetilde{F}_{4}=\mathrm{d} C_{3}+C_{1} \wedge H_{3}+\frac{F_{0}}{2} B \wedge B$, that satisfies the Bianchi identity $\mathrm{d} \widetilde{F}_{4}=H_{3} \wedge \widetilde{F}_{2}$. The first type of charge is given by the integral over the space $\mathcal{M}^{5}$ such that $\partial \mathcal{M}^{5}=\Sigma^{4}$ of the current

$$
\star \mathcal{J}_{D 4}=\mathrm{d} \widetilde{F}_{4}-H_{3} \wedge \widetilde{F}_{2}
$$

that is

$$
Q_{D 4}=\frac{1}{8 \pi^{3} \alpha^{\prime 3 / 2}} \int_{\mathcal{M}^{5}} \star \mathcal{J}_{D 4},
$$

and this charge is gauge invariant but it is not quantized.

Similarly, we can define charges ${ }^{3}$ for the D2-branes and D6-branes, through the following currents

$$
\begin{aligned}
& \star \mathcal{J}_{D 6}=\mathrm{d} \widetilde{F}_{2}-\widetilde{F}_{0} H_{3} \Rightarrow Q_{D 6}=\frac{1}{2 \pi \alpha^{\prime 1 / 2}} \int_{\mathcal{M}^{3}} \star \mathcal{J}_{D 6} \\
& \star \mathcal{J}_{D 2}=\mathrm{d} \widetilde{F}_{6}-H_{3} \wedge \widetilde{F}_{4} \Rightarrow Q_{D 2}=\frac{1}{32 \pi^{5} \alpha^{\prime 5 / 2}} \int_{\mathcal{M}^{7}} \star \mathcal{J}_{D 2},
\end{aligned}
$$

where $\widetilde{F}_{6}=-\star \widetilde{F}_{4}$.

We can define another important charge by regarding the right hand side of the equation

$$
\mathrm{d} \widetilde{F}=H_{3} \wedge \widetilde{F}
$$

as a source for the field strength $\widetilde{F}$, so that

$$
\mathrm{d} \widetilde{F}_{8-p}=\star \mathcal{J}_{\text {Dp }}^{\text {Maxwell }} .
$$

\footnotetext{
${ }^{3}$ Defined as $\left(2 \pi \ell_{s}\right)^{7-p} Q_{\mathrm{Dp}}=\int_{\mathcal{M}^{9-p}} \star \mathcal{J}_{\mathrm{Dp}}$.
} 
Integrating this equation and using the Stokes theorem, with the appropriate normalization, we find

$$
Q_{\mathrm{Dp}}^{\mathrm{Maxwell}}=\frac{1}{\left(2 \pi \ell_{s}\right)^{7-p}} \int_{\Sigma^{8-p}} \widetilde{F}_{8-p}
$$

In the Guarino-Jafferis-Varela (GJV) solution, we can easily see that

$$
n_{6}=Q_{D 2}^{\mathrm{Maxwell}}-\frac{1}{\left(2 \pi \ell_{s}\right)^{5}} \int_{\mathcal{S} S^{5}}\left(B \wedge \mathrm{d} C_{(3)}+\frac{\widetilde{F}_{(0)}}{6} B \wedge B \wedge B\right)
$$

and using the previous results, we find

$$
Q_{D 2}^{\mathrm{Maxwell}}=\frac{81 \sqrt{6} \pi^{3} e^{\phi_{0} / 4} L^{5}(4-\pi)}{\left(2 \pi \ell_{s}\right)^{5}}
$$

Under this perspective, there is one more relevant cycle we may consider in the GJV solution, namely, the 4 -cycle parametrized by the coordinates $\Sigma^{4}=(\lambda, \theta, \phi, \sigma)$. We can easily see that this cycle is the $\mathbb{C P}^{2}$ metric multiplied by the factor $e^{\phi / 2} \Delta \Xi$ that vanishes at the points $\alpha=0, \pi$, and since $\left.\widetilde{F}_{4}\right|_{\Sigma^{4}}$ also vanishes at these points, we conclude that the Page charge $n_{4}$ is zero in this cycle. On the other hand, the Maxwell charge is not trivial, that is

$$
\begin{aligned}
Q_{D 4}^{\text {Maxwell }} & =\frac{1}{\left(2 \pi \ell_{s}\right)^{3}} \int_{\Sigma^{4}} \widetilde{F}_{4} \\
& =\frac{3 \sqrt{3}}{8 \pi} \frac{L^{3} e^{-\phi_{0} / 4}}{\ell_{s}^{3}} \frac{(7+3 \cos 2 \alpha)}{(3+\cos 2 \alpha)^{2}} \sin ^{4} \alpha .
\end{aligned}
$$

Furthermore, the Page charges are well defined up to a large gauge transformations, that shifts the Kalb-Ramond flux

$$
b=\frac{1}{2 \pi \ell_{s}} \int_{\Sigma^{2}} B
$$

by an integer. In fact, since $\mathrm{d} \widetilde{F}_{2}=\widetilde{F}_{0} H_{3}$, we can write

$$
B=\frac{\widetilde{F}_{2}}{\widetilde{F}_{0}}+\mathcal{B}
$$

where $\mathcal{B}=-\mathrm{d} C_{1} / \widetilde{F}_{0}$ and we can easily verify that $\widetilde{F}_{0} \mathcal{B}=-\widehat{F}_{2}$, so in principle it should be quantized, but once we consider a 2-cycle $\Sigma^{2}$ with legs in the coordinate $\alpha$, the integral $\int_{\Sigma^{2}} \mathrm{~d} C_{1}$, vanishes trivially, as well as the Maxwell charge associated to this cycle [24-27]. In particular, we can write the Kalb-Ramond flux as

$$
b=\frac{2 \pi \ell_{s}}{n_{0}}\left(n_{2}+Q_{D 6}^{\mathrm{Maxwell}}\right)
$$

and if the Maxwell charge $Q_{D 6}^{\text {Maxwell }}$ vanishes, the flux $b$ goes with $n_{2} / n_{0}$. 


\section{$2.2 \quad$ Field theory dual}

The field theory dual to this gravitational solution was proposed to be the maximal $\mathcal{N}=8$ SYM theory in $2+1$ dimensions with a single gauge group $\mathrm{SU}(N)$, the $N$ corresponding to the integer in the quantized flux on $S^{6}$ (the Page charge $n_{6}$ of the previous subsection), deformed by Chern-Simons terms with level $k$ related to the mass parameter $m$ by the relation

$$
m=\frac{k}{2 \pi \ell_{s}} .
$$

As we saw, $k$ is the same as the Page charge $n_{0}$ of the previous subsection.

The field theory has 7 adjoint scalars (corresponding to the 7 transverse coordinates to the $2+1$ dimensional worldvolume in 10 dimensional string theory), and 8 fermions transforming under the R-symmetry group $\mathrm{SO}(7)$. At low energy, an adjoint scalar dual to a photon completes the fundamental representation of the full $\mathrm{SO}(8) \mathrm{R}$-symmetry group.

The addition of the Chern-Simons term

$$
\frac{k}{4 \pi} \operatorname{Tr}\left[A \wedge F+\frac{2}{3} A \wedge A \wedge A\right]
$$

together with additional couplings, can preserve $\mathcal{N}=2$ supersymmetry, like the gravitational solution. The SYM theory in 3 dimensions has a dimensionful coupling, but the theory flows in the IR to a fixed point with conformal symmetry, corresponding to the warped $A d S_{4}$ factor in the gravity dual. In this fixed point, the effective 't Hooft coupling is

$$
\lambda=\frac{N}{k},
$$

which must be $\gg 1$ for the supergravity approximation to hold in the gravity dual. Indeed, the curvature in string units is large only if $N / k \gg 1$, as can be see from (2.11).

In $\mathcal{N}=2$ notation, we have an adjoint vector multiplet (that contains a real scalar and a complex fermion) and 3 chiral multiplets with complex scalars $Z, W, T$, like the dimensional reduction of the $3+1$ dimensional $\mathcal{N}=4 \mathrm{SYM}$, with the same superpotential,

$$
W=g \operatorname{Tr}(Z[W, T])
$$

but where $g$ has dimension $1 / 2$, so in the IR we must have $\left[\phi_{i}, \phi_{j}\right]=0$. There is also a conformal potential term for the scalars

$$
V_{c}=\frac{4 \pi^{2}}{k^{2}} \operatorname{Tr}\left(\left[\left[\bar{\phi}^{i}, \phi^{i}\right], \bar{\phi}^{k}\right]\left[\left[\bar{\phi}^{j}, \phi^{j}\right], \phi^{k}\right]\right) .
$$

The theory has R-symmetry $\mathrm{U}(1)$ and flavor symmetry $\mathrm{SU}(3)$ rotating the 3 complex scalars in the chiral multiplets.

The symmetries correspond to the symmetries of the gravity solution. The would-be $\mathrm{SO}(7)$ symmetry of the $S^{6}$ is also broken by the squashing/fibration to $\mathrm{SU}(3)$, acting on $\mathbb{C P}^{2}$, times $\mathrm{U}(1)$, translating $\psi$, or rather $\eta$. 


\section{Particle-like branes and their field theory duals}

In this section we perform a qualitative analysis of branes wrapped on cycles, that look like particles from the point of view of the field theory, and their dual intepretation. We will study static D-branes and moving D-branes.

The D-brane action is inversely proportional to the string coupling. If $g_{s}$ would get large, we would have light D-brane states. But in fact, in [28], the authors argued that massive type IIA theory cannot be strongly coupled. In the examples considered in their work, the authors showed that the string coupling $g_{s}$ has two regimes. In the first regime, $g_{s}$ increases with $N$ as usual in the massless theories, but then it reaches a second regime where it decreases for large $N$.

In the GJV solution there is no such phase transition [17], since the coupling $g_{s}^{\alpha}$ remains bounded,

$$
g_{s}^{\alpha}=e^{\phi} \sim \frac{1}{k^{5 / 6} N^{1 / 6}}<\frac{\ell_{s}}{L_{\text {string }}}, \quad \forall N, k,
$$

where from (2.11), $L_{\text {string }} \sim N^{1 / 6} k^{-1 / 6} \ell_{s}$ (see also (2.12)), and moreover as we said, must be taken to be $\gg \ell_{s}$ for validity of the supergravity approximation, by imposing $N \gg k$.

\subsection{Branes on cycles in the GJV geometry}

In this subsection we consider static branes, that will correspond to solitonic operators in the dual field theory.

We can write the action for a general D p-brane as the sum of the DBI term and a WZ term,

$$
S_{\mathrm{Dp}}=-T_{\mathrm{Dp}} \int \mathrm{d}^{p+1} \xi e^{-\phi} \sqrt{-\operatorname{det}(G+\mathcal{F})}-T_{\mathrm{Dp}} \int \sum_{\tilde{p}} C_{\tilde{p}} \wedge e^{\mathcal{F}},
$$

where $\mathcal{F}=B+2 \pi \alpha^{\prime} F$ and $T_{\mathrm{Dp}}^{-1}=(2 \pi)^{p} \ell_{s}^{p+1}$. It has been shown in [29] that in the presence of Romans mass, we need to add the WZ term for $F_{0}$,

$$
\begin{aligned}
S_{m}^{D p} & =\left.T_{\mathrm{Dp}} F_{0} \int \sum_{r=0}\left(2 \pi \alpha^{\prime}\right)^{r} \frac{\omega_{2 r+1}^{0}}{(r+1) !}\right|_{p+1} \\
& =\left.\frac{k T}{(2 \pi)^{p} \alpha^{\prime p / 2}} \int \sum_{r=0}\left(2 \pi \alpha^{\prime}\right)^{r} \frac{\omega_{2 r+1}^{0}}{(r+1) !}\right|_{p+1},
\end{aligned}
$$

where $\omega_{2 r+1}^{0}$ is a Chern-Simons form, such that $\mathrm{d} \omega_{2 r+1}^{0}=\operatorname{tr}(F)^{r+1} \Rightarrow \omega_{2 r+1}^{0}=\operatorname{tr}\left(A \wedge F^{r}\right)$, and in the second expression we have used that $F_{0}=n_{0} T \sqrt{\alpha^{\prime}} \equiv k T \sqrt{\alpha^{\prime}}$, where $T=$ $1 /\left(2 \pi \alpha^{\prime}\right)$ is the string tension and $F=d A$. We consider just an abelian $(\mathrm{U}(1))$ field strength $F$.

D0-branes. We start with the analysis of D0-branes, which therefore need not be wrapped on cycles, since they are already particle-like. The D0 brane action develops a tadpole from the WZ term (3.3), namely

$$
S_{m}^{D 0}=-T_{D 0} F_{0} \int_{\mathbb{R}} \mathrm{d} t A_{t}=-n_{0} T \int \mathrm{d} t A_{t} .
$$


That means that we need to add $k$ fundamental strings ending on the D0-brane. In principle, there is also the leading term in the WZ part, $\int_{\Sigma} C_{1}$, but since $C_{1}$ has no component along $d t$, corresponding to $\Sigma=\mathbb{R}_{t}$, for a static D0-brane as we consider here, this contribution vanishes.

Moreover, from the DBI action

$$
S_{\mathrm{DBI}}=-T_{D 0} \int_{\mathbb{R}} \mathrm{d} t e^{-\phi} \sqrt{\left|G_{t t}\right|}
$$

we easily see that the brane mass ${ }^{4}$ is

$$
M_{D 0}(\alpha)=\frac{T_{D 0}}{g_{s}^{\alpha}} \sim T_{D 0}\left(N k^{5}\right)^{1 / 6} \gamma(\alpha)^{-1} .
$$

Since as we saw, the string coupling $g_{s}^{\alpha}$ is maximal at $\alpha=\pi / 2$, where it takes the value $e^{\phi_{0}} \sqrt{2}$, the D0-brane will stabilize at this value of $\alpha$, that minimizes its energy, giving

$$
M_{D 0}=T_{D 0} k\left(\frac{N}{k}\right)^{1 / 6} \frac{3^{1 / 6}}{2^{17 / 12} \pi^{1 / 2} \sqrt{\pi}} .
$$

In conclusion, this D0-brane needs $k$ fundamental strings to end on it.

D6-branes. The D6-brane wrapping the whole internal space $\mathcal{S} S^{5}$ develops a tadpole due to the WZ coupling

$$
S_{D 6}^{\mathrm{WZ}}=-T_{D 6} \int_{\mathbb{R} \times \mathcal{S} \mathbb{S} \not} \widehat{F}_{6} \wedge A=-N T \int_{\mathbb{R}} \mathrm{d} t A_{t},
$$

thus we need $N$ fundamental strings ending on this brane. The leading WZ coupling for the D6-brane would come from $C_{7}$ integrated over the compact space, where $\tilde{F}_{8}=$ $d C_{7}-H_{3} \wedge C_{5}+\ldots=-e^{\phi / 2} \tilde{F}_{2}$, but since $\tilde{F}_{2}$ has only components in the compact dimensions, $\tilde{F}_{8}$ is nonzero only when it has at least two non-compact directions. The same is true for the terms $H_{3} \wedge C_{5}+\ldots$, which means that the integral of $C_{7}$ over the compact space and time is zero.

The mass of the D6-brane is then given by

$$
M_{D 6}=T_{D 6} \int \mathrm{d}^{6} \xi e^{-\phi} \sqrt{G_{6}}=\sqrt{\frac{3}{2}} \pi^{3} T_{D 6} \int_{0}^{\pi} \mathrm{d} \alpha \frac{\sqrt{\Omega} \Xi^{2} L_{\text {string }}^{6}}{e^{\phi}} \sim\left(T_{D 6} \alpha^{\prime 3}\right) \frac{N^{7 / 6}}{k^{1 / 6}},
$$

where $G_{6}$ is the determinant of the internal manifold in (2.1a). More precisely, one obtains (also taking into account that $T_{D 6}\left(2 \pi \sqrt{\alpha^{\prime}}\right)^{6}=T_{D 0}$ )

$$
M_{D 6}=T_{D 0} N\left(\frac{N}{k}\right)^{1 / 6} \frac{3^{5 / 3}}{2^{59 / 12} \sqrt{\pi}} \int_{0}^{\pi} d \alpha \sin ^{5} \alpha \frac{(5+\cos 2 \alpha)^{1 / 4}}{3+\cos 2 \alpha} .
$$

An observation about the calculation in (3.9) is that the warp factor $L_{\text {string }}$ is $\alpha$ dependent, and since we still have one factor $L_{\text {string }}$ from the component $G_{t t}$ in the metric, the Dirac-Born-Infeld is not the product of (3.9) and the time integration of $\sqrt{G_{t t}}$. Moreover, for the sake of simplicity, we have ignored the $B$-field.

\footnotetext{
${ }^{4}$ Given a static D $p$-brane wrapping a $p$-cycle $\Sigma$ with a vanishing WZ term, its mass is given by the DBI term, $M_{\mathrm{Dp}}=T_{\mathrm{Dp}} \int_{\Sigma} g_{s}^{-1} \sqrt{\operatorname{det}_{\Sigma}(G+\mathcal{F})}$, where $\operatorname{det}_{\Sigma}$ means the determinant along the $p$-cycle $\Sigma$.
} 
Bound state of D0-branes and D6-branes. Considering a bound state consisting on $p$ D0-branes and $q$ D6-branes, the tadpole is

$$
(p k+q N) \int A
$$

and this term vanishes when $p=-\frac{N}{k} q$. The leading term in the WZ piece is zero, as we just saw, so the mass of this system is

$$
M_{D 0-D 6}=\sqrt{p^{2} M_{D 0}^{2}+q^{2} M_{D 6}^{2}} .
$$

In the case of $p \sim q$ and $N \gg k$ needed for the validity of the supergravity approximation, we can write

$$
M_{D 0-D 6} \simeq q M_{D 6} \sim q \frac{L_{\text {string }}^{6}}{g_{s}} \propto q N\left(\frac{N}{k}\right)^{1 / 6} .
$$

But in general, the formula is symmetric under the interchange of $M_{D 0}$ with $M_{D 6}$ and $p$ with $q$.

D2-branes and D4-branes. In addition, we see that D2-branes and D4-branes are tadpole free, since if we consider that D2-branes on $\mathbb{C P}^{1} \subset \mathbb{C P}^{2}$ and D4-branes on $\mathbb{C P}^{2}$, the $\alpha$-dependent Wess-Zumino terms

$$
\int_{\mathbb{C P}^{1} \times \mathbb{R}_{t}} \mathrm{~d} C_{1} \wedge A \quad \text { and } \quad \int_{\mathbb{C P}^{2} \times \mathbb{R}_{t}} \mathrm{~d} C_{3} \wedge A
$$

will be zero at the minimum. Indeed,

$$
\begin{aligned}
& \int_{\mathbb{C P}^{1}} d C_{1}=a_{0}(\alpha) \int_{\mathbb{C P}^{1}} 2 \mathcal{J}=-2 \pi a_{0}(\alpha) \propto \sin ^{2} \alpha \cos \alpha \\
& \int_{\mathbb{C P}^{2}} d C_{3}=12 \sqrt{3} L^{3} e^{-\phi_{0} / 4} \frac{\sin ^{4} \alpha}{3+\cos 2 \alpha} \int_{\mathbb{C P}^{2}} \mathcal{J} \wedge \mathcal{J} \propto \sin ^{4} \alpha,
\end{aligned}
$$

so are both minimized by $\alpha=0$, where they take the value zero. In the absence of magnetic flux, these are the only tadpoles that could appear.

The leading term in the $\mathrm{WZ}$ piece for the D2-brane, coming from $\int C_{3}$, is trivially zero, but can be made nonzero by considering a moving trajectory, $\eta=\eta(t)$ (so that the rest is $\left.\int_{\mathbb{C P}^{1}} \mathcal{J}=-\pi\right)$. The leading term in the WZ piece for the D4-brane, coming from $\int C_{5}$, is also trivially zero, but can be made nonzero by a moving trajectory, again $\eta=\eta(t$ ) (or rather, $\psi=\psi(t))$. We will analyze these later.

Branes with worldvolume magnetic flux. Instead, we can consider a worldvolume magnetic flux given by $F=\mathcal{N} \mathcal{J}$ in $S^{2} \equiv \mathbb{C P}^{1} \in \mathbb{C P}^{2}$, where $\mathcal{J}$ is the Kähler form of $\mathbb{C P}^{2}$, at fixed $\lambda$, and $\mathcal{N} \in 2 \mathbb{Z}$, see [30-32]. For now, we ignore the subtle issue of the Freed-Witten anomaly [33, 34].

The D2-brane wrapping $\mathbb{C P}^{1}$, for any $\alpha$ (including $\alpha=0$ ), develops the coupling (from the $\int F_{0} \wedge F \wedge A \mathrm{WZ}$ term)

$$
-\frac{k \mathcal{N}}{2} T \int_{\mathbb{R}} \mathrm{d} t A_{t},
$$


where we have used the fact that $\int \mathcal{J}=-\pi$ in $\mathbb{C P}^{1}$, so that now $k \mathcal{N} / 2$ fundamental strings are required to cancel this tadpole.

The magnetic flux also induces a dissolved D0-brane charge of $\mathcal{N}$ from the usual WZ coupling

$$
T_{D 2} \int_{\mathbb{C P}^{1}} F \wedge \int_{\mathbb{R}_{t}} C_{1}=-\mathcal{N} T \int_{\mathbb{R}_{t}} C_{1}
$$

Moreover, the DBI term in the mass of the D2-brane wrapping the squashed space $\widetilde{\mathbb{C P}}^{1}$ is

$$
\begin{aligned}
M_{D 2}(\alpha) & =\frac{T_{D 2}}{g_{s}^{\alpha}} \int_{\widetilde{\mathbb{P}}^{1}} \sqrt{\operatorname{det}\left(G_{\alpha \beta}+2 \pi \alpha^{\prime} F_{\alpha \beta}\right)} \\
& =\frac{\pi T_{D 2}}{2 g_{s}^{\alpha}} \int_{0}^{\pi} \mathrm{d} \theta \sqrt{\left[\left(\Xi^{2}-\Xi \Omega\right) L_{\text {string }}^{4}+\left(\pi \alpha^{\prime} \mathcal{N}\right)^{2}\right] \sin ^{2} \theta+L_{\text {string }}^{4} \Xi \Omega}
\end{aligned}
$$

which is an elliptic integral. For the sake of simplicity, we ignore $\Omega$, then

$$
\begin{aligned}
M_{D 2}(\alpha) & =\frac{T_{D 2}}{g_{s}^{\alpha}} \int_{\mathbb{C P}^{1}} \sqrt{\operatorname{det}\left(G_{\alpha \beta}+2 \pi \alpha^{\prime} F_{\alpha \beta}\right)} \\
& =\frac{\pi T_{D 2}}{g_{s}^{\alpha}}\left(\Xi^{2} L_{\text {string }}^{4}+\left(\pi \alpha^{\prime} \mathcal{N}\right)^{2}\right)^{1 / 2}
\end{aligned}
$$

and we see that the mass of the brane has the usual term related to the brane wrapping an internal cycle, and a shift related to the magnetic charge $\mathcal{N}$. The first (flux-independent) term is proportional to

$$
\sin ^{4} \alpha(5+\cos 2 \alpha)^{1 / 4},
$$

so at $\mathcal{N}=0$, the whole mass (WZ term plus DBI) is minimized by $\alpha=0$, at value $M=0$. The second term however, is proportional to

$$
\frac{3+\cos 2 \alpha}{(5+\cos 2 \alpha)^{3 / 4}}
$$

which is minimal at $\alpha=\pi / 2$ and maximal at $\alpha=0$. Together, at $\mathcal{N} \neq 0$, the mass of the D2-brane (DBI plus vanishing WZ term) is minimized at $\alpha \neq 0$, for a nonzero mass.

The D4-brane wrapping the $\mathbb{C P}^{2}$ cycle at nonzero magnetic flux has a tadpole coming from

$$
-\frac{k \mathcal{N}^{2}}{8} T \int_{\mathbb{R}} \mathrm{d} t A_{t}
$$

and we need $k \mathcal{N}^{2} / 8$ fundamental strings to cancel this tadpole. Observe that now we cannot guarantee that $k \mathcal{N}^{2} / 8$ is an integer, since $\mathcal{N}^{2} \in 4 \mathbb{Z}$. Similar to what occurs in the analysis [31], this suggests that a fractional number of fundamental strings should be added to the D4-brane worldvolume to cancel this tadpole. Moreover, we have a shift in the value of the D4-brane mass due to the magnetic field $F=\mathcal{N} \mathcal{J}$, giving

$$
M_{D 4}(\alpha)=\frac{\pi^{2} T_{D 4}}{2 g_{s}^{\alpha}}\left(\Xi^{2} L_{\text {string }}^{4}+\left(\pi \alpha^{\prime} \mathcal{N}\right)^{2}\right)
$$


where we have deliberately ignored the squashed term $\Omega$ in the metric. ${ }^{5}$ Exactly the same comments as for the D2-brane case apply for the minimization with respect to $\alpha$.

The D4-brane on $\mathbb{C P}^{2}$ also has an induced dissolved D2-brane charge coming from the WZ term

$$
S=2 \pi T_{D 4} \int_{\mathbb{R}_{t} \times \mathbb{C P}^{2}} C_{3} \wedge F=\frac{\mathcal{N}}{2} T_{D 2} \int C_{3}
$$

and a dissolved D0-brane charge coming from the WZ term

$$
S=\frac{1}{2}(2 \pi)^{2} T_{D 4} \int_{\mathbb{R}_{t} \times \mathbb{C P}^{2}}=\frac{\mathcal{N}^{2}}{8} D_{D 0} \int_{\mathbb{R}_{t}} C_{1}
$$

Finally, the D6-brane does not have any tadpole related to the Romans mass $F_{0}$, since $\mathcal{J} \wedge \mathcal{J} \wedge \mathcal{J}=0$. The reader should compare this result with [31], where the Romans mass contributes to the tadpole in a deformation of the ABJM theory [35]. On the other hand, the DBI contribution to the mass of the D6-brane is given by

$$
M_{D 6}=T_{D 6} \int \mathrm{d}^{6} \xi e^{-\phi} L_{\text {string }}^{2} \frac{\sqrt{\operatorname{det}\left(G_{\left.\mathcal{S} S^{5}\right)}\right.}}{\Xi^{2}}\left(L_{\text {string }}^{4} \Xi^{2}+\left(\pi \alpha^{\prime} \mathcal{N}\right)^{2}\right),
$$

where $\operatorname{det}\left(G_{\mathcal{S} S^{5}}\right)$ is the determinant $(2.1 \mathrm{~d})$, so

$$
\begin{aligned}
M_{D 6} & =\frac{\sqrt{3}}{8 \sqrt{2}} T_{D 6} \int \mathrm{d}^{6} \xi e^{-\phi} \sqrt{\Omega} L_{\text {string }}^{2} \cos \lambda \sin ^{3} \lambda \sin \theta\left(L_{\text {string }}^{4} \Xi^{2}+\left(\pi \alpha^{\prime} \mathcal{N}\right)^{2}\right) \\
& =\sqrt{\frac{3}{2}} \pi^{3} T_{D 6} \int_{0}^{\pi} \mathrm{d} \alpha \frac{\sqrt{\Omega} L_{\text {string }}^{2}}{g_{s}^{\alpha}}\left(L_{\text {string }}^{4} \Xi^{2}+\left(\pi \alpha^{\prime} \mathcal{N}\right)^{2}\right)
\end{aligned}
$$

and we see that the magnetic flux shifts the mass of the D6-brane wrapping the internal manifold. Moreover, now we have an integration over $\alpha$, so we don't need to minimize with respect to it. The contribution of the WZ term $\int C_{7}$, where $\tilde{F}_{8}=-e^{\phi / 2} \tilde{F}_{2}$, is zero, since $C_{7}$ doesn't have a term that covers the whole compact space (and time). Therefore the mass is given entirely by the DBI term.

In this case again there are induced dissolved D-brane charges, D2-brane charge coming from

$$
\frac{1}{2} T_{D 6} \int C_{3} \wedge F \wedge F
$$

but no D0-brane charges, since

$$
T_{D 6} \int C_{1} \wedge F \wedge F \wedge F=0
$$

gives zero $(\mathcal{J} \wedge \mathcal{J} \wedge \mathcal{J}=0)$.

\footnotetext{
${ }^{5}$ In fact, the complete expression has the square root of the following determinant

$$
\begin{aligned}
\operatorname{det}\left(G+2 \pi \alpha^{\prime} F\right)= & \frac{1}{128} \sin ^{2} \theta \sin ^{6} \lambda\left(L_{\text {string }}^{4} \Xi^{2}+\pi^{2} \alpha^{\prime 2} \mathcal{N}^{2}\right) \times \\
& \times\left[\cos 2 \lambda\left(L_{\text {string }}^{4} \Xi(\Xi-\Omega)+\pi^{2} \alpha^{\prime 2} \mathcal{N}^{2}\right)+L_{\text {string }}^{4} \Xi(\Xi+\Omega)+\pi^{2} \alpha^{\prime 2} \mathcal{N}^{2}\right] .
\end{aligned}
$$
}




\begin{tabular}{|l|c|c|}
\hline Dp-branes & Mass $M_{\text {Dp }}$ & $q=\#$ Strings \\
\hline D0-branes & $T_{D 0} / g_{s}^{\alpha}$ & $k$ \\
\hline D2-branes & $\pi T_{D 2}\left(\Xi^{2} L_{\text {string }}^{4}+\left(\pi \alpha^{\prime} \mathcal{N}\right)^{2}\right)^{1 / 2} / g_{s}^{\alpha}$ & $k \mathcal{N} / 2$ \\
\hline D4-branes & $\pi^{2} T_{D 4}\left(\Xi^{2} L_{\text {string }}^{4}+\left(\pi \alpha^{\prime} \mathcal{N}\right)^{2}\right) / 2 g_{s}^{\alpha}$ & $k \mathcal{N}^{2} / 8$ \\
\hline D6-branes & $\sqrt{3 / 2} \pi^{3} T_{D 6} / g_{s} \int \mathrm{d} \alpha \frac{\sqrt{\Omega} L_{\text {string }}^{2}}{\gamma(\alpha)}\left(L_{\text {string }}^{4} \Xi^{2}+\left(\pi \alpha^{\prime} \mathcal{N}\right)^{2}\right)$ & $N$ \\
\hline
\end{tabular}

Table 1. Branes configurations.

\subsection{Field theory dual: baryon operators}

We would like to understand what these particle-like branes with strings ending on them mean from the point of view of the dual field theory.

We start by summarizing the results for the various branes we have found, in table 1 .

The static solitonic branes with strings attached to them correspond to baryon vertex operators (when introducing external quarks in the theory), as understood first by Witten [36] in the context of the D5-brane wrapping $S^{5}$ in $A d S_{5} \times S^{5}$ for $\mathcal{N}=4$ SYM. More precisely, in that case for the gauge group $\mathrm{SU}(N)$, one could construct the baryon

$$
B^{I_{1} \ldots I_{N}}=\epsilon_{i_{1} \ldots i_{N}} q^{i_{1} I_{1}} \ldots q^{i_{N} I_{N}}
$$

and the external (heavy) quarks $q^{i_{k} I_{k}}$ correspond to long strings coming from the boundary and reaching the brane. The mass of the brane (3.10) corresponds to the mass of the baryon with the quark masses subtracted, i.e. the baryon vertex operator.

The field theory dual to the GJV solution is also a $\mathrm{SU}(N)$ SYM theory, though now with a CS term at level $k$. As such, we again expect the presence of the same baryon vertex constructed using the $\epsilon_{i_{1} \ldots i_{N}}$ tensor. We indeed find this from the D6-brane wrapped over the compact space, which indeed corresponds in the T-dual type IIB theory to the D5-brane wrapped over the compact space.

The D2-brane and D4-brane baryons are harder to understand, but they appear only in the presence of magnetic flux, so they represent some interesting dynamical objects, that should be studied further.

Also the D0-branes, giving a baryon made up of $k$ external quarks, are harder to understand from the point of view of the $\mathrm{SU}(N)$ gauge theory. But it is known that many Chern-Simons gauge theories have level-rank duality, as reviewed, derived and extended in the recent paper [37]. In certain cases, an $\mathrm{SU}(N)_{k}$ theory is dual to an $\mathrm{SU}(k)_{N}$ theory [38, 39], or even in the case of $\mathrm{U}(N)_{k}$ we can have a duality to $\mathrm{U}(k)_{N}$ [40]. In [37], a duality valid in general was proposed between a $\mathrm{SU}(N)$ theory at level $k$ and an $\mathrm{U}(k)$ theory at level $-N$.

In our case, the theory contains other fields besides the gauge fields, and also a SYM term, not just the CS term (the SYM term however is subdominant to the CS term at low energies), so the physics is not very clear, but we would expect from the level-rank dual formulation to have at the very least a baryon constructed with the $\epsilon_{i_{1} \ldots i_{k}}$ tensor. Moreover, the mass of this baryon vertex must match (3.7). An interesting observation is that both (3.7) and (3.10) are proportional to $T_{D 0}(N / k)^{1 / 6}=T_{D 0} \lambda^{1 / 6}$, whereas the 
D0-brane mass is proportional to $k$ and the D6-brane mass with $N$. This suggests that level-rank duality certainly plays a role, in exchanging the $N$ and $k$ prefactors, whereas the coupling dependence stays the same $\lambda^{1 / 6}$, and is not inverted as naive replacing of $N$ with $k$ would suggest.

Finally, we have also bound states of the D0-branes and D6-branes, giving new bound states of the two types of baryon vertices. Again from the point of view of the field theory, we have evidence of level-rank duality, when we exchange $N$ with $k, p$ with $q$, while leaving $\lambda=N / k$ invariant, as seen in (3.12). The case of $p=-\frac{N}{k} q$ (the minus sign signifies considering anti-baryons) is very interesting: in this case there is no tadpole, so it would seem like we don't need external quarks, but rather have a solitonic object composed of only glue, and yet with the symmetry properties of a combination of baryons. Unlike the case of a usual $N$-baryon/ $N$-antibaryon combination, where the result would be annihilation, in this case the bound state would seem to be stable, which is quite unusual.

We see that we have strong evidence for level-rank duality in the field theory and, since such a relation is expected for our theory, this also is strong evidence for the correct identification of the field theory dual to the gravitational GJV solution.

Another observation is that it is expected that the external magnetic flux breaks the level-rank duality, and indeed we see that the D2 and D4 baryonic vertices are not level-rank dual to each other (or to themselves).

\subsection{Brane stability and operator bounds}

Now we try to proceed as in $[30,31]$ and analyze the dynamical stability of the brane configurations of subsection (3.1). This will give bounds on the magnetic fluxes, that can be reinterpreted as bounds on the dissolved charges (since those depend only on $\mathcal{N}$, as we saw).

We want to consider the system of a D $p$-brane wrapping the $p$-cycle and $q$ fundamental strings necessary to cancel the tadpole, that is

$$
S=S_{\mathrm{Dp}}+S_{q F S}
$$

where we consider the string at fixed $\alpha$, defined by the minimum in the mass of the $\mathrm{D} p$ brane, $\alpha_{\min } \cdot{ }^{6}$

$$
\begin{gathered}
S_{\mathrm{Dp}}=-M_{\mathrm{Dp}} \int \mathrm{d} t L_{\text {string }} u \equiv-M_{\mathrm{Dp}}^{L} \int \mathrm{d} t u \\
S_{q F S}=-\left.q T L_{\text {string }}^{2}\right|_{\alpha, \text { min. }} \int \mathrm{d} x \mathrm{~d} t \sqrt{u^{4}+u^{\prime 2}}
\end{gathered}
$$

and we have considered a string configuration with $x=-\sigma, t=\tau$ and $u=u(\sigma)$. There are two contributions to the equations of motion of this system, one from the bulk and another from the boundary [41-43]. These equations are, respectively,

$$
\begin{aligned}
\partial_{\sigma} \frac{\partial \mathcal{L}}{\partial x^{\prime}}=0 & \Rightarrow \frac{u^{4}}{\sqrt{u^{4}+u^{\prime 2}}}=c_{0} \\
\text { Boundary } & \Rightarrow \frac{u_{0}^{\prime}}{\sqrt{u_{0}^{4} x_{0}^{\prime 2}+u_{0}^{\prime 2}}}=\frac{M_{\mathrm{Dp}}^{L}}{L_{\text {string }, \alpha, \min .}^{2} q T} .
\end{aligned}
$$

\footnotetext{
${ }^{6}$ In the D6-brane case, the extra $L_{\text {string }}$ must be included in the integral over $\alpha$ defined in $M_{D 6}$.
} 
We define

$$
\sqrt{1-\beta^{2}} \equiv \frac{M_{\mathrm{Dp}}^{L}}{L_{\text {string }, \alpha, \min , q T}^{2} q} \Rightarrow \beta=\sqrt{1-\left(\frac{M_{\mathrm{Dp}}^{L}}{L_{\text {string }, \alpha, \min .}^{2} q T}\right)^{2}},
$$

so that we write the equations of motion for the $\mathrm{D} p$-brane q-strings as one single equation

$$
\frac{u^{4}}{\sqrt{u^{4}+u^{\prime 2}}}=\beta u_{0}^{2} .
$$

Integrating this equation, we find the size of the brane configuration,

$$
l_{w b}=\frac{1}{u_{0}} \int_{1}^{\infty} \mathrm{d} y \frac{\beta}{y^{2} \sqrt{y^{4}-\beta^{2}}},
$$

where $y=u / u_{0}$. This equation is similar to the equations in [30,31], and the integral is solved in terms of hypergeometric functions.

D0-brane. Consider first the D0-brane. Reality of the function (3.32) implies

$$
0<\frac{M_{D 0}^{L}}{L_{\text {string }, \alpha, \min .} q T}=\frac{2 \pi \ell_{s}}{g_{s, \text { min. }}^{\alpha} k L_{\text {string }, \alpha, \min .}}<1
$$

but this is always satisfied, because from (3.1) we have

$$
1>\frac{\ell_{s}}{g_{s}^{\alpha} L_{\text {string }, \alpha}}>\frac{2 \pi \ell_{s}}{g_{s}^{\alpha} L_{\text {string, }, \alpha}} .
$$

Consider next D2-branes and D4-branes with worldvolume magnetic flux.

D2-brane. Neglect the squashed term $\Omega$ in the metric. For the D2-brane, with $q=k \mathcal{N} / 2$, reality of $\beta$ in (3.32) implies

$$
\frac{M_{D 2}}{L_{\text {string }, \alpha, \min . T}}<\frac{k \mathcal{N}}{2},
$$

which gives, since $\pi T_{D 2} / T=1 /\left(4 \pi \alpha^{\prime}\right)$,

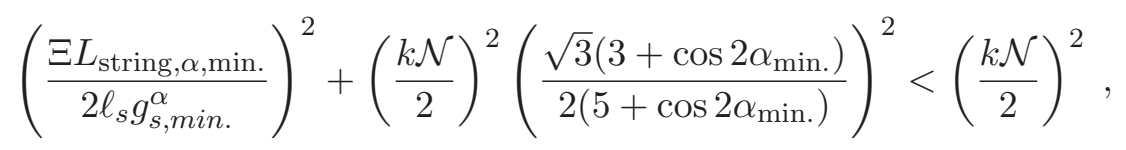

which is a lower bound on $q=k \mathcal{N} / 2$, or (at fixed $k$ ) on the dissolved charge $\mathcal{N}$. Parametrically, we obtain

$$
(k \mathcal{N})^{2}>\mathcal{O}\left[N^{2 / 3} k^{4 / 3}\right] \Rightarrow \mathcal{N}>\mathcal{O}\left[\left(\frac{N}{k}\right)^{1 / 3}\right] .
$$

In $[30,31]$, the authors showed that the there are stable D2-brane configurations in the presence of magnetic flux $\mathcal{N}$ until some upper bound, interpreted as an upper bound on dissolved charges, related to the stringy exclusion principle. In the case (3.38) however, we have a lower bound, which means that stability of this configurations implies the presence of a minimum magnetic flux on the brane worldvolume. 
D4-brane. A similar situation occurs for the D4-brane, with $q=k \mathcal{N}^{2} / 8$. Stability of the configuration requires that $\beta$ in (3.32) is real, which implies

$$
\frac{M_{D 4}}{L_{\text {string, }, \alpha, \min . T}}<\frac{k \mathcal{N}^{2}}{8} .
$$

This in turn gives

$$
\frac{1}{2}\left[\frac{\Xi^{2} L_{\text {string }, \alpha \text { min. }}^{3} / \ell_{s}^{3}}{8 \pi g_{s}^{\alpha}}+\frac{k \mathcal{N}^{2}}{8} \frac{\sqrt{3}\left(3+\cos 2 \alpha_{\text {min. }}\right)}{2\left(5+\cos 2 \alpha_{\text {min. }}\right)}\right]<\frac{k \mathcal{N}^{2}}{8},
$$

which is again a lower bound on $q=k \mathcal{N}^{2} / 8$, or (at fixed $\mathrm{k}$ ) on the dissolved D0-brane charge $\mathcal{N}^{2} / 8$. It is then also a lower bound on the magnetic flux on the worldvolume that makes the D4-brane stable, parametrically also

$$
k \mathcal{N}^{2}>\mathcal{O}\left[N^{2 / 3} k^{1 / 3}\right] \Rightarrow \mathcal{N}>\mathcal{O}\left[\left(\frac{N}{k}\right)^{1 / 3}\right]
$$

D6-brane. Finally, we consider the case of D6-branes, with $q=N$. In this case there is a small difference: the coordinate $\alpha$ is integrated over on the D6-brane worldvolume, but the strings need to be at given $\alpha$. Minimizing their action means that their $L_{\text {string }}^{2}$ factor must be minimum, i.e. for $\alpha=\pi / 2$, when

$$
L_{\text {string }}(\pi / 2) g_{s}=\frac{2 \pi \ell_{s}}{k} \sqrt{\frac{2}{3}} .
$$

The stability bound is again the reality of $\beta$, which gives

$$
\frac{M_{D 6}^{L}}{L_{\text {string }}^{2}(\pi / 2) T}=\frac{M_{D 6}}{L_{\text {string }}(\pi / 2) T}<N,
$$

and is rewritten as

$$
\frac{k}{\left(2 \pi \ell_{s}\right)^{6}} \pi^{3} \int d \alpha \frac{\sqrt{\Omega} L_{\text {string }}^{2}}{\gamma(\alpha)}\left[L^{4} \Xi^{2}+\left(\pi \ell_{s} \mathcal{N}\right)^{2}\right]<N .
$$

This is now really an upper bound on the magnetic flux $\mathcal{N}$, or more precisely on the induced dissolved D2-brane charge $\sim \mathcal{N}^{2}$, just like in [30,31]. In order of magnitude, we obtain

$$
k\left[\mathcal{O}\left(\frac{N}{k}\right)+\mathcal{N}^{2} \mathcal{O}\left(\left(\frac{N}{k}\right)^{1 / 3}\right)\right]<N \Rightarrow \mathcal{N}^{2}<\mathcal{O}\left[\left(\frac{N}{k}\right)^{2 / 3}\right] .
$$

In conclusion, we see that D0-branes are always stable, but the regions of stability of the D2-branes and D4-branes, and of the D6-branes are parametrically opposite: $\mathcal{N}>$ $\mathcal{O}\left[(N / k)^{1 / 3}\right]$ and $\mathcal{N}<\mathcal{O}\left[(N / k)^{1 / 3}\right]$, respectively. There might be a window for all of them to be stable, depending on the exact numerical factors, which one would have to compute numerically. 


\subsection{Reducing the number of fundamental strings}

Following $[32,41]$ we can consider a configuration with a reduced number $r$ of fundamental strings going to the boundary, the rest of the strings (up to the number required to cancel the tadpole) can go to the IR of the gravity solution. In holographic terms, this means that the baryons we consider have less quarks than the baryons of the previous subsections.

Using the conventions of [32], the configuration we consider consists of a $\mathrm{D} p$-brane wrapped on a $p$-cycle and located at $u=u_{0}$ in the radial direction. In addition, we consider $l$ strings stretching from $u_{0}$ to the boundary of AdS4 (parametrized in polar coordinates) and $(q-l)$ straight strings stretching from $u_{0}$ to $u=0$. The action is given by

$$
S=S_{\mathrm{DBI}}+S_{l F S}+S_{(q-l) F S}
$$

where

$$
\begin{aligned}
S_{\mathrm{DBI}}^{D p} & =-M_{\mathrm{Dp}}^{L} \int \mathrm{d} t u \\
S_{l F 1} & =-l T_{F 1} L_{\text {string }}^{2} \int \mathrm{d} t \mathrm{~d} u \sqrt{1+u^{4} r^{\prime 2}} \\
S_{(q-l) F 1} & =-(q-l) T_{F 1} L_{\text {string }}^{2} \int \mathrm{d} t \mathrm{~d} u .
\end{aligned}
$$

The equation of motion for the $l$ strings implies that

$$
r^{\prime}=\frac{\tilde{u}^{2}}{u^{2} \sqrt{u^{4}-\tilde{u}^{4}}},
$$

where $0 \leq \tilde{u} \leq u_{0}$ is the turning point of each string, and we see that at the turning point $r^{\prime} \rightarrow \infty$. Moreover, as in [32], the boundary equation can be written as

$$
\frac{1}{a} \sqrt{1-\tilde{\beta}^{2}}+\frac{1}{a}(1-a)=\sqrt{1-\frac{\tilde{u}^{4}}{u_{0}^{4}}} \leq 1
$$

where $a=l / q$ and

$$
\sqrt{1-\tilde{\beta}^{2}}=\frac{M_{\mathrm{Dp}}^{L}}{L_{\text {string }}^{2} T_{F 1} q} \leq 1,
$$

and this bound must be satisfied in order to get a stable configuration. Moreover, from the boundary equation (3.50) we conclude that

$$
l \geq \frac{q}{2}\left(\sqrt{1-\tilde{\beta}^{2}}+1\right)
$$

and this bound defines the minimum value of quarks to create a stable baryon in the field theory. Furthermore, from the table 1 we see that fundamental strings attached to D2-branes and D4-branes are possible just in the presence of magnetic field, therefore, the condition (3.52) also depends on the magnitude of the magnetic field on the brane

worldvolume. We can easily see that for $\beta=0$, that occurs when $M_{\mathrm{Dp}}^{L}=L_{\text {string }}^{2} T_{F 1} q$, the 
quark configurations are made of $q$-quarks, and the results reduce to what we obtained in the previous subsection.

Moreover, using (3.49) we can easily show that the size of this configuration is

$$
l_{w b}=\frac{\tilde{u}^{2}}{u_{0}^{3}} \int_{1}^{\infty} \mathrm{d} z \frac{1}{z^{2} \sqrt{z^{4}-\frac{\tilde{u}^{4}}{u_{0}^{4}}}},
$$

where $z=u / u_{0}$, and similarly to (3.34), this result can be integrated in terms of hypergeometric functions, giving as there

$$
l_{w b}=\frac{\tilde{u}^{2}}{u_{0}^{3}}{ }^{2} F_{1}\left(\frac{1}{2}, \frac{3}{4}, \frac{7}{4} ; \frac{\tilde{u}^{4}}{u_{0}^{4}}\right) .
$$

\subsection{Giant gravitons: gravity calculation}

Giant gravitons in a gravitational solution correspond to solitonic states, obtained by wrapping D-branes on cycles in the geometry, which are therefore extended states, that are moving in a direction transverse to the cycle, and that are degenerate in energies with graviton states. A proper treatment of the giant gravitons will take a separate paper, so here we will just sketch the main points.

The GJV gravity solution with metric (2.1a) is a solution of massive type IIA string theory, and we saw that there are wrapped D2-branes and D4-branes that are tadpole-free (in the absence of magnetic flux), thus could be interpreted as giant gravitons. Moreover, we have already said that we could obtain a nonzero mass (by a nonzero WZ term) for a moving brane, whereas the static brane will have vanishing mass. This is exactly the giant graviton case.

1. We can obtain a standard kind of giant graviton by wrapping a D2-brane on an $S^{2}$. The $A d S_{4}$ metric to be considered is

$$
\mathrm{d} s_{A d S_{4}}^{2}=-\left(1+r^{2}\right) \mathrm{d} t^{2}+\frac{\mathrm{d} r^{2}}{1+r^{2}}+r^{2} \mathrm{~d} \Omega_{2}^{2},
$$

where $\mathrm{d} \Omega_{2}^{2}=\mathrm{d} \vartheta^{2}+\sin ^{2} \vartheta \mathrm{d} \varphi^{2}$ parametrizes the two sphere $S^{2}$, on which the D2-brane is wrapped.

2. Another type of giant graviton is obtained by wrapping a D2-brane on an $\mathbb{C P}^{1}=S^{2}$ inside the compact geometry. We can consider the $S^{2}=\mathbb{C P}^{1} \subset \mathbb{C P}^{2}$ defined by $\mathcal{J}$. By considering the leading WZ term $\int C_{3}$ in the case when $\eta=\eta(t)$, we will obtain a nonzero contribution to the mass, proportional to

$$
\frac{\sin ^{4} \alpha}{3+\cos 2 \alpha} \dot{\eta}
$$

but the DBI term for the moving brane will also be modified.

3. Yet another type of giant graviton will be obtained by considering D4-branes wrapping the $\mathbb{C P}^{2}$. Again, the leading WZ term in $\int C 5$ for $\eta=\eta(t)$ will give a nonzero contribution to the mass (note that $\tilde{F}_{6}=-e^{\phi / 2} * \tilde{F}_{4}$ ), 


\subsubsection{D2-brane on $S^{2} \subset A d S_{4}$}

We consider the metric (3.55) for the $A d S_{4}$ space, with the sphere $S^{2}$ parametrized by $(\vartheta, \varphi)$. The ansatz for the giant gravitons is given by

$$
\xi^{0}=t, \xi^{1}=\vartheta, \xi^{2}=\varphi, \psi=\psi(t)
$$

and we set $\lambda=\pi / 2$ and $\alpha=\pi / 2$. We study the giant gravitons dynamics following the well known recipe [44-46], in particular, giant gravitons in the GJV solution are similar to the configurations considered in the ABJM case [47-49]. The induced metric on the brane is

$$
\mathrm{d} s_{D 2}^{2}=L_{\text {string }}^{2}\left[\left(\frac{9}{4} \dot{\psi}^{2}-\left(1+r^{2}\right)\right) \mathrm{d} t^{2}+r^{2} \mathrm{~d} \Omega_{2}^{2}\right]
$$

and the DBI plus WZ action is

$$
S_{D 2}=-4 \pi T_{D 2} \frac{L_{\text {string }}^{3}}{g_{s}^{\alpha}} \int \mathrm{d} t\left(r^{2} \sqrt{1+r^{2}-\dot{\chi}^{2}}-r^{3}\right)
$$

where we have defined $\chi \equiv \frac{3}{2} \psi$. When we write the momentum $P_{\chi}$ as

$$
P_{\chi}=\frac{4 \pi T_{D 2} L_{\text {string }}^{3}}{g_{s}^{\alpha}} \frac{r^{2} \dot{\chi}}{\sqrt{1+r^{2}-\dot{\chi}^{2}}} \equiv \frac{4 \pi T_{D 2} L_{\text {string }}^{3}}{g_{s}^{\alpha}} p
$$

and the Hamiltonian is simply

$$
\mathcal{H}=\frac{4 \pi T_{D 2} L_{\text {string }}^{3}}{g_{s}^{\alpha}}\left(\sqrt{\left(1+r^{2}\right)\left(p^{2}+r^{4}\right)}-r^{3}\right)
$$

and this functional has two minima, with $\mathcal{H}=P_{\chi}$, namely $r=0, p$ and the latter we call giant graviton. Furthermore, if we insert the giant graviton into (3.60) we find $\chi=t$, so that the angular velocity $\dot{\chi}$ equals the speed of light.

As a technical detail, one may notice that the giant gravitons in $[47,48]$ move in a circle $S^{1} \subset \mathbb{C P}^{3}$ parametrized by the coordinate $\sigma$ in the Fubini-Study metric

$$
\begin{aligned}
\mathrm{d} s_{\mathbb{C P}^{3}}= & \mathrm{d} \lambda^{2}+\frac{1}{4} \sin ^{2} \lambda\left(\mathrm{d} \theta^{2}+\sin ^{2} \theta \mathrm{d} \phi^{2}\right)+\frac{1}{4} \cos ^{2} \lambda\left(\mathrm{d} \tilde{\theta}^{2}+\sin ^{2} \tilde{\theta} \mathrm{d} \tilde{\phi}^{2}\right) \\
& +\frac{1}{4} \cos ^{2} \lambda \sin ^{2} \lambda(\mathrm{d} \sigma+\cos \theta \mathrm{d} \phi-\cos \tilde{\theta} \mathrm{d} \tilde{\phi})
\end{aligned}
$$

that has a subspace $\mathbb{C P}^{2}$ for constant $(\tilde{\theta}, \tilde{\phi})$. Therefore, in order to have a proper identification of the giant graviton of this section with the giants of $[47,48]$, we must consider the motion confined in the coordinate $\sigma$, instead of $\psi$, in squashed 6-sphere of the GJV solution. The derivation is isomorphic to what we have done so far, so we will not repeat the calculations. 


\subsubsection{D2-brane on the squashed $S^{2} \subset \mathcal{S} S^{5}$}

When we consider that the D2-branes wraps a two sphere inside the internal space $\mathcal{S} S^{5}$ defined by (2.1c), the calculations are more involved because of the non-diagonal terms in the metric, but the idea is essentially the same, with one remarkable difference, the role of the angular coordinates, as we will see below.

The ansatz is given by

$$
\xi^{0}=t, \xi^{1}=\theta, \xi^{2}=\phi, \psi=\psi(t),
$$

and we set $r=0$ and constant $\lambda$ and $\sigma$. The induced metric on the brane is

$$
\begin{aligned}
\mathrm{d} s_{D 2}^{2}=L_{\text {string }}^{2} & {\left[\left(\Omega \dot{\psi}^{2}-1\right) \mathrm{d} t^{2}+\frac{\Xi \sin ^{2} \lambda}{4} \mathrm{~d} \theta^{2}+2 \Omega \frac{\dot{\psi} \sin ^{2} \lambda \cos \theta}{2} \mathrm{~d} t \mathrm{~d} \phi\right.} \\
& \left.+\frac{\sin ^{2} \lambda}{4}\left[\Xi\left(\sin ^{2} \theta+\cos ^{2} \lambda \cos ^{2} \theta\right)+\Omega \sin ^{2} \lambda \cos ^{2} \theta\right] \mathrm{d} \phi^{2}\right],
\end{aligned}
$$

and the Dirac-Born-Infeld action reads

$$
\begin{aligned}
S_{\mathrm{DBI}}= & -\frac{T_{D 2} L_{\text {string }}^{3} \pi}{2 g_{s}^{\alpha}} \int \mathrm{d} \theta \mathrm{d} t \sin ^{2} \lambda \sqrt{\Xi\left(\Xi \cos ^{2} \theta \cos ^{2} \lambda+\Xi \sin ^{2} \theta+\Omega \cos ^{2} \theta \sin ^{2} \lambda\right)} \times \\
& \times \sqrt{1-\frac{\left(\Xi \cos ^{2} \theta \cos ^{2} \lambda+\Xi \sin ^{2} \theta-3 \Omega \cos ^{2} \theta \sin ^{2} \lambda\right)}{\left(\Xi \cos ^{2} \theta \cos ^{2} \lambda+\Xi \sin ^{2} \theta+\Omega \cos ^{2} \theta \sin ^{2} \lambda\right)}} .
\end{aligned}
$$

The $\theta$-integration can be carried out easily and gives an expression in terms of elliptic functions. But in order to avoid unnecessary complications, we will keep it as it is. Together with the Wess-Zumino term

$$
S_{\mathrm{WZ}}=-\frac{T_{D 2} L_{\text {string }}^{3} \pi}{g_{s}^{\alpha}} \int \mathrm{d} \theta \sin \theta \int \mathrm{d} t \sin ^{2} \lambda \frac{3 \sqrt{3}}{(3+\cos 2 \alpha)^{2}} \sin ^{4} \alpha \dot{\psi},
$$

we have the D2-brane action

$$
S_{D 2}=-\int \mathrm{d} \theta \int \mathrm{d} t\left(f(\alpha, \lambda ; \theta) \sqrt{1-g(\alpha, \lambda ; \theta) \dot{\psi}^{2}}-h(\alpha, \lambda ; \theta) \dot{\psi}\right)
$$

where we write the action in terms of the functions $f(\alpha, \lambda ; \theta), g(\alpha, \lambda ; \theta)$ and $h(\alpha ; \theta)$ given by

$$
\begin{aligned}
f(\alpha, \lambda ; \theta) & =\frac{T_{D 2} L_{\text {string }}^{3} \pi}{2 g_{s}^{\alpha}} \sin ^{2} \lambda \sqrt{\Xi\left(\Xi \cos ^{2} \theta \cos ^{2} \lambda+\Xi \sin ^{2} \theta+\Omega \cos ^{2} \theta \sin ^{2} \lambda\right)} \\
& \equiv f_{0}(\alpha) \sin ^{2} \lambda \sqrt{f_{1}(\alpha ; \theta) \cos ^{2} \lambda+f_{2}(\alpha ; \theta)} \\
g(\alpha, \lambda ; \theta) & =\frac{\Omega\left(\Xi \cos ^{2} \theta \cos ^{2} \lambda+\Xi \sin ^{2} \theta-3 \Omega \cos ^{2} \theta \sin ^{2} \lambda\right)}{\left(\Xi \cos ^{2} \theta \cos ^{2} \lambda+\Xi \sin ^{2} \theta+\Omega \cos ^{2} \theta \sin ^{2} \lambda\right)} \\
& \equiv \frac{g_{1}(\alpha ; \theta) \cos ^{2} \lambda+g_{2}(\alpha ; \theta)}{f_{1}(\alpha ; \theta) \cos ^{2} \lambda+f_{2}(\alpha ; \theta)} \\
h(\alpha, \lambda ; \theta) & =-\frac{T_{D 2} \pi L_{\text {string }}^{3} \sin \theta}{g_{s}^{\alpha}} \frac{3 \sqrt{3}}{(3+\cos 2 \alpha)^{2}} \sin ^{4} \alpha \sin ^{2} \lambda \equiv h_{1}(\alpha ; \theta) \sin ^{2} \lambda .
\end{aligned}
$$


For the sake of simplicity, we assume that $\lambda=\pi / 2$.

With these definitions, the momentum (up to a $\theta$-integration) is

$$
P_{\psi}=\frac{g f \dot{\psi}}{\sqrt{1-g \dot{\psi}^{2}}}+h
$$

which we can invert to write $\dot{\psi}$ in terms of $P_{\psi}$, as

$$
\dot{\psi}=\frac{P_{\psi}-h}{\sqrt{(f g)^{2}+g\left[P_{\psi}-h\right]^{2}}} .
$$

The Hamiltonian becomes

$$
\begin{aligned}
\mathcal{H} & =P_{\psi} \dot{\psi}-\mathcal{L} \\
& =\frac{1}{g} \sqrt{g\left(P_{\psi}-h\right)^{2}+f^{2} g^{2}} .
\end{aligned}
$$

Writing down explicitly the equation $\partial_{\alpha} \mathcal{H} \equiv \mathcal{H}^{\prime}=0$, we have

$$
-2 g^{\prime} \mathcal{H}^{2}+\frac{1}{g}\left[g^{\prime}\left(2 f^{2} g+\left(P_{\psi}-h\right)^{2}\right)-2 g\left(P_{\psi}-h\right) h^{\prime}+2 g^{2} f f^{\prime}\right]=0,
$$

and solving the condition for the angle $\alpha$ that follow from this equation, we find the pointlike and giant gravitons solutions.

The solution that corresponds to the pointlike graviton is given by $\sin \alpha \cos \alpha=0$. Since $\alpha=0, \pi$ are isolated conical singularities, the system is not well defined at these points. We set $\alpha=\pi / 2$ for the pointlike gravitons. For the giant graviton solution, we must solve the equation $\partial_{\alpha} \mathcal{H}=0$, for $\alpha \neq \pi / 2$, that gives an algebraic equation which must be solved for $X=\sin ^{2} \alpha$.

The non-diagonal terms in the metric make the explicit verification of these algebraic conditions very involved. For simplicity then, we consider a dielectric brane, such that the electric field is conveniently chosen to cancel the non-diagonal terms of the metric [50]. In this somewhat contrived case, the D2-brane action (3.67) reduces to

$$
S_{D 2}=-\int \mathrm{d} \theta \int \mathrm{d} t\left(f(\alpha, \lambda ; \theta) \sqrt{1-\Omega \dot{\psi}^{2}}-h(\alpha, \lambda ; \theta) \dot{\psi}\right)
$$

with momentum

$$
P_{\psi}=\frac{f \Omega \dot{\psi}}{\sqrt{1-\Omega \dot{\psi}^{2}}}+h \Rightarrow \dot{\psi}=\frac{P_{\psi}-h}{\sqrt{(f \Omega)^{2}+\Omega\left(P_{\psi}-h\right)^{2}}},
$$

and energy given by

$$
\mathcal{H}=\frac{1}{\Omega} \sqrt{(f \Omega)^{2}+\Omega\left(P_{\psi}-h\right)^{2}},
$$

that is equivalent to the replacement $g \rightarrow \Omega$ in the Hamiltonian (3.73). In this case the pointlike gravitons have again $\alpha=\pi / 2$. 


\subsubsection{D4-brane on the squashed $\mathbb{C P}^{2} \subset \mathcal{S} S^{5}$}

Finally, we consider a D4-brane wrapping the whole squashed complex projective space $\mathbb{C P}^{2}$, so the induced metric on the D4-brane is

$$
\begin{aligned}
\mathrm{d} s_{D 4}^{2}=L_{\text {string }}^{2}\{ & \left(-1+\Omega \dot{\psi}^{2}\right) \mathrm{d} t^{2}+2 \frac{\Omega \dot{\psi} \sin ^{2} \lambda}{2}(\mathrm{~d} \sigma+\cos \theta \mathrm{d} \phi) \mathrm{d} t+\Xi \frac{\sin ^{2} \lambda}{4} \mathrm{~d} \theta^{2}+\Xi \mathrm{d} \lambda^{2} \\
& \left.+\frac{\sin ^{2} \lambda}{4}\left[\Xi \sin ^{2} \theta \mathrm{d} \phi^{2}+\left(\Xi \cos ^{2} \lambda+\Omega \sin ^{2} \lambda\right)(\mathrm{d} \sigma+\cos \theta \mathrm{d} \phi)^{2}\right]\right\} .
\end{aligned}
$$

It gives the Dirac-Born-Infeld action

$$
S_{\mathrm{DBI}}=-\tilde{f}(\alpha) \int \mathrm{d} t \mathrm{~d} \lambda \sin ^{3} \lambda \sqrt{\Omega(1-\cos 2 \lambda)+\Xi\left(1-\Omega \dot{\psi}^{2}\right)(1+\cos 2 \lambda)},
$$

where

$$
\tilde{f}(\alpha)=T_{D 4} \frac{\sqrt{2} L_{\mathrm{string}}^{5} \pi^{2} \Xi^{3 / 2}}{g_{s}^{\alpha}} .
$$

Given the 6-form RR field $\widetilde{F}_{6}$ defined by $\widetilde{F}_{6}=-e^{\phi / 2} * \widetilde{F}_{4}$, its restriction to $\mathcal{S} S^{5}$ is

$$
\left.\widetilde{F}_{6}\right|_{\mathcal{S} S^{5}}=-\left(\frac{3 L^{3} e^{-\phi_{0} / 4}}{4} \sqrt{\frac{3}{2}} \Delta \sqrt{\Omega} \Xi^{2} e^{\phi / 2} \sin ^{3} \lambda \cos \lambda \sin \theta\right) \mathrm{d} \alpha \mathrm{d} \psi \mathrm{d} \theta \mathrm{d} \lambda \mathrm{d} \phi \mathrm{d} \sigma
$$

where the wedge product of forms is implicit. We may write this expression as

$$
\left.\widetilde{F}_{6}\right|_{\mathcal{S} S^{5}}=\mathrm{d} C_{5}^{\mathcal{S} S^{5}}
$$

where

$$
C_{5}^{\mathcal{S} S^{5}}=-\left(81 L^{5} e^{\phi_{0} / 4} \sqrt{\frac{3}{2}} A(\alpha) \sin ^{3} \lambda \cos \lambda \sin \theta\right) \mathrm{d} \psi \mathrm{d} \theta \mathrm{d} \lambda \mathrm{d} \phi \mathrm{d} \sigma
$$

and

$$
A(\alpha)=\int \mathrm{d} \alpha \frac{\sin ^{5} \alpha}{(3+\cos 2 \alpha)^{2}}=\frac{1}{2} \arctan (\cos \alpha)-\frac{\cos \alpha}{4}-\frac{\cos \alpha}{3+\cos 2 \alpha} .
$$

Therefore, the Wess-Zumino term for a D4-brane wrapping the squashed space $\mathbb{C P}^{2}$ is

$$
S_{\mathrm{WZ}}=-T_{D 4} \int C_{5}^{\mathcal{S} S^{5}}=\tilde{g}(\alpha) \int \mathrm{d} t \mathrm{~d} \lambda \sin ^{3} \lambda \cos \lambda \dot{\psi}
$$

where we have defined $\tilde{g}(\alpha)=324 T_{D 4} \sqrt{6} \pi^{2} e^{\phi_{0} / 4} A(\alpha)$. Finally, the D4-brane action is simply

$$
\begin{gathered}
S_{D 4}=-\int \mathrm{d} t \mathrm{~d} \lambda\left(\tilde{f}(\alpha) \sin ^{3} \lambda \sqrt{\Omega(1-\cos 2 \lambda)+\Xi\left(1-\Omega \dot{\psi}^{2}\right)(1+\cos 2 \lambda)}\right. \\
\left.-\tilde{g}(\alpha) \sin ^{3} \lambda \cos \lambda \dot{\psi}\right) .
\end{gathered}
$$


We leave the $\lambda$-integral untouched, since the result is quite complicated. Therefore, the Hamiltonian (up to a $\lambda$-integration) is

$$
\begin{aligned}
\mathcal{H}= & \frac{\sqrt{\Xi(1+\cos 2 \lambda)+\Omega(1-\cos 2 \lambda)}}{\Xi \Omega(1+\cos 2 \lambda)} \times \\
& \times \sqrt{\tilde{f}^{2} \sin ^{2} \lambda \Xi^{2} \Omega^{2}(1+\cos 2 \lambda)^{2}+\Xi \Omega\left(P_{\psi}-\tilde{g} \sin ^{3} \lambda \cos \lambda\right)^{2}},
\end{aligned}
$$

and again, studying the condition that follows from $\partial_{\alpha} \mathcal{H}=0$ we find the pointlike and giant graviton solutions.

\subsection{Giant gravitons: field theory operators}

As usual, the field theory operators corresponding to the giant gravitons are subdeterminants, of the type

$$
\epsilon_{a_{1} \ldots a_{k} a_{k+1} \ldots a_{N}} \epsilon^{b_{1} \ldots b_{k} a_{k+1} \ldots a_{N}}\left(Z_{b_{1}}^{a_{1}} \ldots Z_{b_{k}}^{a_{k}}\right)
$$

or with $W$ and $T$ replacing any of the $Z$ fields. More generally, the operators are Schur polynomials for some complicated reprentation of the symmetry group.

-For the D2-brane wrapping $S^{2} \subset \mathbb{C P}^{2}$, the fields inside the bracket are $Z$ 's and $W$ 's in an $\mathrm{SU}(2)$ invariant combination, forming representations of $\mathrm{SU}(2)$.

-For the D2-brane wrapping $S^{2} \subset A d S_{4}$, we should replace the $Z$ 's with $D_{a} Z$ 's, where $a$ corresponds to the two angles on $\Omega_{2}$, the spatial 2-sphere in $A d S_{4}$. Again, the combinations of $D_{a} Z$ 's should be in some representation of $\mathrm{SU}(2)$.

-For the D4-brane wrapping $\mathbb{C P}^{2}$, we should have all the $Z, W, T$ inside the bracket, transforming under $\mathrm{SU}(3)$ (the symmetry group of $\mathbb{C P}^{2}$ ), i.e. forming a representation of this symmetry group.

\subsection{Gauge coupling from wrapped brane}

One can easily show that given a induced metric on a D $p$-brane wrapping an $n$-cycle $\Sigma^{n}$ of the form,

$$
\mathrm{d} s_{\mathrm{Dp}}^{2}=e^{2 A} \eta_{\mu \nu} \mathrm{d} x^{\mu} \mathrm{d} x^{\nu}+\mathrm{d} s_{\Sigma}^{2},
$$

the gauge coupling defined on the Minkowski worldvolume of the brane is

$$
\frac{1}{g_{Y M}^{2}}=T_{\mathrm{Dp}}\left(2 \pi \alpha^{\prime}\right)^{2} \int_{\Sigma} \mathrm{d}^{n} \xi e^{(3+n-p) A-\phi} \sqrt{\operatorname{det}(G+B)_{a b}}
$$

where $\phi$ is the dilaton and $G_{a b}$ and $B_{a b}$ are the metric and the Kalb-Ramond field along the $n$-cycle $\Sigma^{n}$.

In the GJV solution, we can wrap a probe D4-brane on a 2-cycle parametrized by $(\theta, \phi)$ at $\lambda=0$. In this case, the (3 dimensional) gauge coupling of the probe is given by

$$
\frac{1}{g_{Y M}^{2}}=\frac{\Delta^{3 / 2} \Xi e^{-\phi / 4}}{4 \pi \alpha^{\prime 3 / 2}} \frac{1}{y} \sim \frac{\left(N^{2} k\right)^{1 / 3}}{y}=\frac{N}{\lambda^{1 / 3}} \frac{1}{y},
$$

where we have used the $A d S_{4}$ coordinate system $\mathrm{d} s_{\mathrm{AdS} 4}^{2}=\left(\mathrm{d} y^{2}+\eta_{\mu \nu} \mathrm{d} x^{\mu} \mathrm{d} x^{\nu}\right) / y^{2}$.

We observe that, as expected, the probe gauge coupling is now given by the curvature in string units as $\propto \lambda^{1 / 6}$. 


\section{Strings in the geometry}

\subsection{Wilson loops}

Despite the fact that the Yang-Mills gauge coupling is dimensionful in three dimensions, and that we are studying the gravity dual of a conformal field theory, Wilson loops are interesting observables to consider. Using the standard prescription of $[42,43,51]$ in the metric background (2.1a), we consider a string configuration that consists of an open string with its endpoints attached to the $A d S_{4}$ boundary, $u \rightarrow \infty$, at the points $x=0$ and $x=L_{q \bar{q}}$, and that extends into the bulk and reaches a minimum radial coordinate $u_{0}$ exactly at $x=L_{q \bar{q}} / 2$.

The ansatz for this configuration is given by $t=\tau, x=x(\sigma)$ and $u=u(\sigma)$, so that the Nambu-Goto action is

$$
S=\frac{L_{\text {string }}^{2}}{2 \pi \alpha^{\prime}} \int \mathrm{d} \tau \mathrm{d} \sigma \sqrt{u^{4} x^{\prime 2}+u^{\prime 2}}
$$

where the prime denotes derivative with relation to $\sigma$. From the equations of motion for this system we have

$$
\begin{aligned}
\frac{\mathrm{d} u}{\mathrm{~d} x}=-\frac{u^{2}}{u_{0}^{2}} \sqrt{u^{4}-u_{0}^{4}} \quad \text { for } \quad x<\frac{L_{q \bar{q}}}{2} \\
\frac{\mathrm{d} u}{\mathrm{~d} x}=+\frac{u^{2}}{u_{0}^{2}} \sqrt{u^{4}-u_{0}^{4}} \quad \text { for } \quad x>\frac{L_{q \bar{q}}}{2} .
\end{aligned}
$$

Using these equations, we can determined that the quark-antiquark distance is given by

$$
L_{q \bar{q}}=\frac{2}{u_{0}} \int_{1}^{\infty} \mathrm{d} y \frac{1}{y^{2} \sqrt{y^{4}-1}}=\frac{1}{u_{0}} \frac{(2 \pi)^{3 / 2}}{\Gamma(1 / 4)^{2}} .
$$

where $y=u / u_{0}$.

Furthermore, if we replace this solution, the quark-antiquark potential diverges, so that we renormalize this system by removing the mass of two $W$-bosons, that consists of two strings stretching from the boundary into the bulk of the AdS space. Therefore, we find that the potential is given by

$$
E_{q \bar{q}}=-\frac{(2 \pi)^{2}}{\Gamma(1 / 4)^{4}} \frac{L_{\text {string }}^{2}}{\alpha^{\prime} L_{q \bar{q}}},
$$

which is similar to the result of [42]. It still has the conformal form $\propto 1 / L_{q \bar{q}}$, but there is an interesting difference when we write it in terms of the 't Hooft coupling $\lambda$. In the $A d S_{5} \times S^{5}$ case one obtains a quark-antiquark potential that behaves as the square root of the 't Hooft coupling, $E_{q \bar{q}} \sim \sqrt{\lambda} / L_{q \bar{q}}$, but in our case, since the 't Hooft coupling is $\lambda=N / k$ (see also $[28,35]$ ), we have the one third power of the coupling,

$$
E_{q \bar{q}}=-\frac{2^{13 / 6} \pi^{3}}{3^{2 / 3} \Gamma(1 / 4)^{4}} \frac{\sqrt{5+\cos 2 \alpha} \lambda^{1 / 3}}{L_{q \bar{q}}} \sim \frac{\lambda^{1 / 3}}{L_{q \bar{q}}} .
$$




\subsection{Operators of large spin}

Strings rotating around the circle $S^{2} \in A d S_{4}$ in global coordinates will be dual to high spin operators [52], and the anomalous dimension of these operators in SYM is generically

$$
E-S=f(\lambda) \ln S
$$

We take the $A d S_{4}$ metric as

$$
\mathrm{d} s_{A d S_{4}}^{2}=-\cosh ^{2} \rho \mathrm{d} t+\mathrm{d} \rho^{2}+\sinh ^{2} \rho\left(\mathrm{d} \vartheta^{2}+\sin ^{2} \vartheta \mathrm{d} \varphi^{2}\right)
$$

and we consider that the string is at the equator of $S^{2}, \vartheta=\pi / 2$ and

$$
t=\tau, \rho=\rho(\sigma), \varphi=\omega \tau .
$$

The Nambu-Goto action is then

$$
S_{N G}=-\frac{4 L_{\text {string }}^{2}}{2 \pi \alpha^{\prime}} \int_{0}^{\rho_{0}} \mathrm{~d} \rho \sqrt{\cosh ^{2} \rho-\sinh ^{2} \rho \dot{\varphi}^{2}}
$$

and the 4 comes from the fact that the closed string forms a loop that consists of two parallel segments, and spins as a rigid rod around its center at $\rho=0$ and extends to $\rho_{0}$, determined by $\omega^{2}=\operatorname{coth}^{2} \rho_{0}$ [52]. Similarly, the string energy and spin are

$$
\begin{aligned}
& E=\frac{4 L_{\text {string }}^{2}}{2 \pi \alpha^{\prime}} \int_{0}^{\rho_{0}} \mathrm{~d} \rho \frac{\cosh ^{2} \rho}{\sqrt{\cosh ^{2} \rho-\sinh ^{2} \rho \omega^{2}}} \\
& S=\frac{4 L_{\text {string }}^{2}}{2 \pi \alpha^{\prime}} \int_{0}^{\rho_{0}} \mathrm{~d} \rho \frac{\omega \sinh ^{2} \rho}{\sqrt{\cosh ^{2} \rho-\sinh ^{2} \rho \omega^{2}}},
\end{aligned}
$$

respectively.

Consider now that the string length is much larger than the $A d S_{4}$ radius, $L \sim L_{\text {string }}$, then $S \gg L_{\text {string }}^{2}$, and it corresponds to the limit $\omega \rightarrow 1$. Taking $\omega \approx 1+2 \eta$ for $\eta \ll 1$, one can easily obtain $2 \rho_{0}=\ln (1 / \eta)$ and

$$
E=\frac{L_{\text {string }}^{2}}{2 \pi \alpha^{\prime}}(1 / \eta+\ln (1 / \eta)+\cdots), \quad S=\frac{L_{\text {string }}^{2}}{2 \pi \alpha^{\prime}}(1 / \eta-\ln (1 / \eta)+\cdots),
$$

so that the cusp anomalous dimension is found to be

$$
f(\lambda)=L_{\text {string }}^{2} / \pi \alpha^{\prime} \propto \lambda^{1 / 3},
$$

and the proportionality constants are easily obtained from (2.11). Note that, in contrast to $[2,52]$, the anomalous cusp dimensional scales as $\lambda^{1 / 3}$ instead of $\lambda^{1 / 2}$.

\section{Entanglement entropy}

The metric of the GJV solution (2.1a) has the form of a warped, squashed $A d S_{4} \times S^{6}$. Because of this, it is worth to consider the calculation of the holographic entanglement 
entropy, acording to the prescription of Ryu and Takayanagi [53], extended by Klebanov, Kutasov and Murugan to more general gravity duals in [54].

We consider a $d+2$ dimensional gravity dual, with a $d+1$ dimensional quantum field theory at its boundary, and we want to compute the entanglement resulting from introducing a closed $d-1$ dimensional spatial surface $\partial A$ that separates the spatial region $A$ from the rest of $d$ dimensional space (at constant time). Consider then the $d$ dimensional spatial surface $\gamma$ in the gravity dual that ends on the same boundary surface, $\partial \gamma=\partial A$. Then the holographic entanglement entropy is given by

$$
S_{A}=\frac{1}{4 G_{N}^{(d+2)}} \int_{\gamma} d^{d} \sigma \sqrt{G_{\text {ind }}^{(d)}} .
$$

For a 10 dimensional metric, moreover in the presence of a nontrivial dilaton field $\phi$, we have instead

$$
S_{A}=\frac{1}{4 G_{N}^{(10)}} \int_{\gamma} d^{8} \sigma e^{-2 \phi} \sqrt{G_{\text {ind }}^{(8)}} .
$$

Here $G_{\text {ind }}$ is the induced string frame metric on the surface $\gamma$.

The string frame metric $d s_{\text {string }}^{2}=e^{\phi / 2} d s_{\mathrm{E}}^{2}$ for the GJV solution, (2.1a), is

$$
d s_{\text {string }}^{2}=L^{2} e^{\phi_{0} / 2}(5+\cos \alpha)^{1 / 2}\left[d s_{\left(A d S_{4}\right)}^{2}+\frac{3}{2} d \alpha^{2}+\Xi d s_{\mathbb{C P}^{2}}^{2}+\Omega \eta^{2}\right]
$$

which can be put in the form

$$
\begin{aligned}
d s_{\text {string }}^{2} & =A(z)\left[d s_{\left(A d S_{4}\right)}^{2}+d z^{2}\right]+g_{i j}(z) d \theta^{i} d \theta^{j} \\
& =A(z)\left[\frac{d y^{2}-d t^{2}+d x_{1}^{2}+d x_{2}^{2}}{y^{2}}+d z^{2}\right]+g_{i j}(z, \ldots) d \theta^{i} d \theta^{j}
\end{aligned}
$$

where $z=\alpha \sqrt{3 / 2}$ and $A(z)=L^{2} e^{\phi_{0} / 2}(5+\cos \alpha)^{1 / 2}$.

This type of metric is somewhat more general than metrics considered before, for instance than in [54].

Consider the region $A$ to be the interval $(-l, l)$ in one of the spatial directions, called $x$, times the full space in the others, so that $\partial A$ are the two endpoins of the interval, times the full space in the other directions. The surface $\gamma$ in the gravity dual is then a curve $y(x)$ with boundary conditions $y(-l)=0=y(l)$, where $y$ is the radial coordinate of AdS. This is the same as in the simple $A d S_{d+2} / C F T_{d+1}$ case. In our $A d S_{4}$ case, $d=2$, and we can take $x_{1}=x, x_{2}$ being trivial, but the discussion is more general than this $d=2$ case.

The induced metric on $\gamma($ with $y=y(x))$ is then in this more general case (with $d-1$ coordinates $\vec{x}$ and one $x$ )

$$
d s_{\text {string }}^{2}=A(z)\left[\frac{d x^{2}\left(1+y^{\prime 2}\right)+d \vec{x}^{2}}{y^{2}}+d z^{2}\right]+g_{i j}(z, \ldots) d \theta^{i} d \theta^{j} .
$$

Denoting

$$
V_{\mathrm{int}}(z) \equiv d^{D-d-3} \theta \int \sqrt{\operatorname{det} g_{i j}}
$$


the entanglement entropy of the interval becomes

$$
\begin{aligned}
S_{A} & =\frac{1}{4 G_{N}^{(10)}} \int d^{d-1} \vec{x} \int d z \int_{-l}^{l} d x e^{-2 \phi} V_{\mathrm{int}}(z) y(x)^{-d} A(z)^{\frac{d+1}{2}} \\
& =\left\{\frac{1}{4 G_{N}^{(10)}}\left[\int d z e^{-2 \phi(z)} V_{\mathrm{int}}(z) A(z)^{\frac{d+1}{2}}\right]\right\} \int d^{d-1} \vec{x} \int_{-l}^{+l} d x y(x)^{-d} \sqrt{1+\left(y^{\prime}(x)\right)^{2}}
\end{aligned}
$$

The square brackets offer a constant renormalization of $G_{N}^{(10)}$, but otherwise the result is the same as for $A d S_{d+2}=A d S_{4}$. The same profile for $y(x)$ is obtained, and the same final result for the entanglement entropy as a function of $l$.

\section{Giant magnons}

We now turn to some large string theory objects, specifically giant magnons, that come from long (macroscopic) strings in the gravity dual, and correspond in the gauge theory to spin chain states of large momentum.

\subsection{Gravity calculation}

\subsubsection{Review of $A d S_{5} \times S^{5}$ giant magnons}

The giant magnons were defined by Hofman and Maldacena in [55]. Since the regular magnons (spin excitations) correspond to quantum states of the string on the pp wave, obtained as a Penrose limit of the gravity dual, giant magnons corespond to classical, i.e. long, strings in the gravity dual. Specifically, since we look for giant magnons in the $\mathrm{SU}(2)=\mathrm{SO}(3)$ sector of the field theory (made up from two complex scalars $Z$ and $W$ ), in the gravity dual the motion of the strings is restricted to $\mathbb{R}_{t} \times S^{2}$. The spatial $S^{2}$ is embedded in the $S^{5}$ of $A d S_{5} \times S^{5}$ as

$$
d s_{S^{5}}^{2}=\sin ^{2} \theta d \phi^{2}+d \theta^{2}+\cos ^{2} \theta d \Omega_{3}^{2},
$$

by $d \Omega_{3}=0$. We consider the gauge $t=\tau, \phi-t=\phi^{\prime} \equiv \sigma$, where $(\tau, \sigma)$ are the string worldsheet coordinates. Then the induced metric on the string worldsheet is

$$
\begin{aligned}
d s_{\text {induced }}^{2} & =-d \tau^{2}-\frac{d s_{S^{5}}^{2}}{d \tau^{2}} d \tau^{2}+\frac{d s_{S^{5}}^{2}}{d \sigma^{2}} d \sigma^{2}+2 d \sigma d \tau \frac{d s_{S^{5}}^{2}}{d \tau d \sigma} \\
& =-\cos ^{2} \theta d \tau^{2}+\left(\sin ^{2} \theta+\theta^{\prime 2}\right) d \sigma^{2}+2 \sin ^{2} \theta d \sigma d \tau .
\end{aligned}
$$

Then the string action is, for $R^{2} /\left(2 \pi \alpha^{\prime}\right)=\sqrt{\lambda}$,

$$
\begin{aligned}
S & =\frac{\sqrt{\lambda}}{2 \pi} \int d \sigma d \tau \sqrt{-\operatorname{det} g_{a b, \text { induced }}} \\
& =\frac{\sqrt{\lambda}}{2 \pi} \int d t \int d \phi^{\prime} \sqrt{\cos ^{2} \theta \theta^{\prime 2}+\sin ^{2} \theta}
\end{aligned}
$$

and its (integrated) equation of motion is

$$
\sin \theta=\frac{\sin \theta_{0}}{\cos \phi^{\prime}}
$$


and $\theta_{0}$ is an integration constant, such that $\phi^{\prime} \in\left[-\left(\pi / 2-\theta_{0}\right),+\left(\pi / 2-\theta_{0}\right)\right]$. This is a string that rotates around $S^{2} \in S^{5}$, while describing an arc that starts and ends on the Equator, with an angle $\Delta \phi=\Delta \phi^{\prime}=2\left(\pi / 2-\theta_{0}\right)$ between the enpoints.

The (lightcone) energy of the string is

$$
\epsilon=E-J=\frac{\sqrt{\lambda}}{\pi}\left|\sin \frac{p}{2}\right| .
$$

\subsubsection{Giant magnons in the gravity dual}

In the GJV gravity dual, we could consider various giant magnon solutions (spinning classical strings). But here we are interested simply in embedding the simple $\mathrm{SU}(2)$-invariant giant magnons from the $A d S_{5} \times S^{5}$ case in the present context. For that, it suffices to find an $S^{2}$ fiber inside the metric (2.1a).

We can write the $\mathbb{C P}^{2}$ metric as (see (A.6))

$$
d s_{\mathbb{C P}^{2}}^{2}=d \lambda^{2}+\frac{1}{4} \sin ^{2} \lambda\left(\Sigma_{1}^{2}+\Sigma_{2}^{2}+\cos ^{2} \lambda \Sigma_{3}^{2}\right)
$$

where the left-invariant one-forms are

$$
\begin{aligned}
& \Sigma_{1}=-\sin \sigma d \theta+\cos \sigma \sin \theta d \phi \\
& \Sigma_{2}=\cos \sigma d \theta+\sin \sigma \sin \theta d \phi \\
& \Sigma_{3}=d \sigma+\cos \theta d \phi .
\end{aligned}
$$

Here $\lambda \in[0, \pi / 2], \sigma \in[0,4 \pi], \theta \in[0, \pi]$ and $\phi \in[0,2 \pi]$. Note that

$$
\Sigma_{1}^{2}+\Sigma_{2}^{2}=d \theta^{2}+\sin ^{2} \theta d \phi^{2},
$$

so this combination forms an $S^{2}$.

We then have two obvious possibilities for $S^{2}$ embedding:

- $\lambda=\pi / 2, \theta=\theta_{0}$, which gives (the range for $\theta$ and $\phi$ is also correct)

$$
d s_{\mathbb{C P}^{2}}^{2} \rightarrow \frac{1}{4}\left(d \theta^{2}+\sin ^{2} \theta d \phi^{2}\right)=\frac{1}{4} d s_{S^{2}}^{2} .
$$

- $\sigma=\sigma_{0}, \phi=\phi_{0}$, which gives

$$
d s_{\mathbb{C P}^{2}}^{2} \rightarrow\left(d \lambda^{2}+\sin ^{2} \lambda(d(\theta / 2))^{2},\right.
$$

however with the range $\theta / 2 \in[0, \pi / 2], \lambda \in[0, \pi / 2]$. Since for the giant magnon solution we would need $\theta / 2$ to be in $[0,2 \pi]$, we could extend the domain by symmetry (the metric is unchanged for the extension).

In the gravity dual, the action will be proportional to

$$
\frac{L_{\text {string }}^{2}}{2 \pi \alpha^{\prime}} \propto\left(\frac{N}{k}\right)^{1 / 3}=\sqrt{\lambda^{2 / 3}},
$$

so we would obtain a giant magnon energy of

$$
\epsilon=E-J \propto \sqrt{\lambda^{2 / 3}}\left|\sin \frac{p}{2}\right| .
$$




\subsection{Field theory}

As we saw, the dual field theory has 3 complex scalars in chiral multiplets, transforming under $\mathrm{SU}(3), Z, W, T$. For the giant magnons, we want to consider an $\mathrm{SU}(2)(=\mathrm{SO}(3))$ sector, that corresponds to the motion on the $S^{2}$ with the same symmetry. We choose $Z$ and another scalar $\Psi$ to be the fields transforming under it.

We consider a spin chain formed from $Z$ and $\Psi$ in the "dilute gas approximation", where the states are formed mostly from $Z$ 's, with just a few $W$ excitations, similar to the BMN construction in $3+1$ dimensions [56]. As there, the ground state is

$$
\left|0, p^{+}\right\rangle=\frac{1}{\sqrt{J} N^{J / 2}} \operatorname{Tr}\left[Z^{J}\right]
$$

We consider a few excitations made by inserting in the l'th position $\Psi e^{\frac{2 \pi i n l}{J}}$ inside the trace. Considering $\{W, T\}=\left\{\Phi^{m}\right\}, m=1,2$, we obtain a priori different spin chains for the $\Psi$ insertion being $\Phi^{m}, \bar{\Phi}^{m}$ or $\bar{Z}$.

Similarly to the case of $3+1$ dimensional $\mathcal{N}=4$ SYM, described in appendix A of [49], but more similar to the ABJM case of the same paper, we obtain a Hamiltonian with eigenenergies of

$$
\epsilon(p)=\sqrt{1+\frac{f_{a}(\lambda)}{\pi^{2}} \sin ^{2} \frac{p}{2}},
$$

where $p=2 \pi n / J$ is the momentum of the giant magnon, and $f_{a}(\lambda)$ is a function that changes its form depending on the $\Psi$ insertion, $\left(\Phi^{m}, \bar{\Phi}^{m}, \bar{Z}\right)$. For a regular magnon with $n \sim 1$, and in the limit $\lambda / J^{2}=$ finite (as $\lambda, J \rightarrow \infty$ ) we would have

$$
\epsilon(p) \sim \sqrt{1+\frac{f_{a}(\lambda) n^{2}}{J^{2}}}
$$

which is the result in the BMN limit, corresponding to the Penrose limit. But if we take instead the limit $\lambda$ fixed and large, $p \sim 1$, we get

$$
\epsilon(p)=\frac{\sqrt{f_{a}(\lambda)}}{\pi}\left|\sin \frac{p}{2}\right|
$$

and from the giant magnon solution in the gravity dual (6.12), we expect $f_{a}(\lambda) \propto \lambda^{2 / 3}$.

\subsubsection{Hamiltonian and diagonalization}

We reproduce some of the steps in appendix A of [49], that give the energies above. The introduction of the various fields inside the trace (which gives a cyclic ordering) can be described by the introduction of Cuntz oscillators $a_{i}, i=1, \ldots, J$ acting on a vacuum, i.e. something like $a^{\dagger} \ldots a^{\dagger} b^{\dagger} \ldots a^{\dagger}|0\rangle$, where $a$ and $b$ correspond to $Z$ and $W$, respectively. The Cuntz oscillators satisfy

$$
a_{i} a_{j}^{\dagger}=\delta_{i j} ; \quad \sum_{i} a_{i}^{\dagger} a_{i}=1-|0\rangle\left\langle 0\left|; \quad a_{i}\right| 0\right\rangle=0 .
$$

If we only have a few "impurities" $W$ inside the trace, we can switch to a description in terms of independent Cuntz oscillators $b_{j}^{\dagger}$ at each site, satisfying

$$
b_{i} b_{i}^{\dagger}=1 ; \quad b_{i}^{\dagger} b_{i}=1-(|0\rangle\langle 0|)_{i} ; \quad b_{i}|0\rangle_{i}=0,
$$


and

$$
\left[b_{i}, b_{j}\right]=\left[b_{i}^{\dagger}, b_{j}\right]=\left[b_{i}^{\dagger}, b_{j}^{\dagger}\right]=0, \quad \forall i \neq j .
$$

Defining the Fourier modes $b_{n}$ by

$$
b_{j}=\frac{1}{\sqrt{J}} \sum_{n=1}^{J} e^{\frac{2 \pi i j n}{J}} b_{n},
$$

and acting only on states in the dilute gas approximation, i.e.

$$
\left|\psi_{\left\{n_{i}\right\}}\right\rangle=|0\rangle_{1} \ldots\left|n_{i_{1}}\right\rangle_{i_{1}} \ldots\left|n_{i_{k}}\right\rangle_{i_{k}} \ldots|0\rangle_{J}
$$

one obtains the commutation relations

$$
\left[b_{n}, b_{m}^{\dagger}\right]\left|\psi_{\left\{n_{i}\right\}}\right\rangle=\left(\delta_{n m}-\frac{1}{J} \sum_{k} e^{\frac{2 \pi i i_{k}(m-n)}{J}}\right)\left|\psi_{\left\{n_{i}\right\}}\right\rangle ; \quad\left[b_{n}, b_{m}\right]=\left[b_{n}^{\dagger}, b_{m}^{\dagger}\right]=0,
$$

so, up to $1 / J$ corrections, the usual oscillator commutation relations.

The interaction term in the Lagrangean in $2+1$ dimensions is

$$
L_{\text {int }}=-\frac{4 \pi^{2}}{k^{2}} \operatorname{Tr}\left(\left[\left[\bar{\Phi}^{i}, \bar{\Phi}^{j}, \bar{\Phi}^{k}\right]\left[\left[\bar{\Phi}^{j}, \Phi^{j}\right], \Phi^{k}\right]\right),\right.
$$

but where in the IR we must impose $\left[\Phi^{i}, \Phi^{j}\right]=0$ from the superpotential term, and it becomes equivalent, under the definition of the discretized field $\phi_{j}=\left(b_{j}+b_{j}^{\dagger}\right) / \sqrt{2}$, to $(\lambda=N / k)$

$$
-2 \lambda^{2} \sum_{j}\left(\phi_{j}-\phi_{j+1}\right)^{2}
$$

Then the total Hamiltonian acting on the states created by Cuntz oscillators, including a free part, is found to be

$$
H=\sum_{j=1}^{J} \frac{b_{j} b_{j}^{\dagger}+b_{j}^{\dagger} b_{j}}{2}+\lambda^{2} \sum_{j=1}^{J}\left(b_{j+1}+b_{j+1}^{\dagger}\right)\left(b_{j}+b_{j}^{\dagger}\right)-\left(b_{j}+b_{j}^{\dagger}\right)^{2},
$$

where $\lambda=g_{Y M}^{2} N$. This Hamiltonian can be diagonalized by the redefinition

$$
b_{n}=\frac{c_{n, 1}+c_{n, 2}}{\sqrt{2}} ; \quad b_{J-n}=\frac{c_{n, 1}-c_{n, 2}}{\sqrt{2}},
$$

followed by the Bogoliubov transformation

$$
\begin{aligned}
\tilde{c}_{n, 1} & =a_{n} c_{n_{1}}+b_{n} c_{n, 1}^{\dagger} \\
\tilde{c}_{n, 2} & =a_{n} c_{n_{1}}-b_{n} c_{n, 1}^{\dagger} \\
a_{n} & =\frac{\left(1+\alpha_{n}\right)^{1 / 4}+\left(1+\alpha_{n}\right)^{-1 / 4}}{2} \\
b_{n} & =\frac{\left(1+\alpha_{n}\right)^{1 / 4}-\left(1+\alpha_{n}\right)^{-1 / 4}}{2} \\
\alpha_{n} & =\lambda^{2}(\cos (2 \pi n / J)-1)=-2 \lambda^{2} \sin ^{2} \frac{\pi n}{J},
\end{aligned}
$$


leading finally to

$$
H=\sum_{n=1}^{J / 2} \omega_{n}\left[\frac{\tilde{c}_{n, 1}^{\dagger} \tilde{c}_{n, 1}+\tilde{c}_{n, 1} \tilde{c}_{n, 1}^{\dagger}}{2}+\frac{\tilde{c}_{n, 2}^{\dagger} \tilde{c}_{n, 2}+\tilde{c}_{n, 2} \tilde{c}_{n, 2}^{\dagger}}{2}\right]
$$

where

$$
\omega_{n}=\sqrt{1+4\left|\alpha_{n}\right|}=\sqrt{1+8 \lambda^{2} \sin ^{2} \frac{\pi n}{J}} .
$$

In this section we have studied giant magnons, but an obvious question is what happens with regular magnons. They will give the usual pp wave (Penrose) limit of the gravity dual. This limit will be studied further in [57].

\section{Conclusions}

In this paper we have studied various observables for the GJV duality between the warped, squashed $A d S_{4} \times S^{6}$ background and the 3 dimensional $\mathcal{N}=2$ SYM-CS theory at level $k$ at the fixed point.

We have found that static wrapped D-branes give baryon vertices. At zero magnetic flux, we have D0-brane and D6-brane baryons, as well as their bound states, and they present evidence for the existence of a level-rank duality: besides the baryon vertex for $N$ quarks we also have a baryon vertex for $k$ quarks, and their masses are proportional to $N$ and $k$, respectively, other than the $\lambda^{1 / 6}$ piece. At nonzero magnetic flux, we have some interesting new baryon vertices that depend on the flux $\mathcal{N}$. Brane probes give a gauge coupling proportional to $\lambda^{1 / 6}$.

Moving wrapped D-branes give giant gravitons, and we have found that we can have a D2-brane wrapped on $\mathbb{C P}^{1}$ or a D4-brane wrapped on $\mathbb{C P}^{2}$, both moving in the transverse $\eta($ or $\psi$ ) direction. They correspond as usual to subdeterminant (or more generally Schur polynomial) operators.

Entanglement entropy, calculated from the holographic prescription generalizing the one of Ryu and Takayanagi, gives nothing new with respect to AdS space, other than a renormalization of the Newton's constant. Wilson loops and the anomalous dimensions of operators of large spin are obtained from long strings in the dual, and both are proportional to $\lambda^{1 / 3}$.

Finally, giant magnons are found, also long strings in an $S^{2}$ subspace of the geometry, that correspond to spin chain operators in the field theory. Their Hamiltonian gives eigenergies consistent with the string calculation, and similar to the 4 dimensional SYM and ABJM cases.

The GJV duality has many more important issues to be studied. A novel one is the level-rank duality discovered here. Others, like the Penrose limit, will be studied elsewhere [57]. It seems like this is just the tip of an iceberg, that should see many new developments in the near future. 


\section{Acknowledgments}

The work of HN is supported in part by CNPq grant 301219/2010-9 and FAPESP grant 2014/18634-9. The work of TA is supported by the Recém Doutor grant from PROPeUNESP. We would like to thank Georgios Itsios and Éoin Ó Colgáin for comments.

\section{A Conventions}

The metric of the space $A d S_{4}$, normalized as $R_{m n}^{A d S_{4}}=-3 g_{m n}^{A d S_{4}}$, is

$$
\begin{aligned}
\mathrm{d} s_{A d S_{4}}^{2} & =\frac{\mathrm{d} u^{2}}{u^{2}}+u^{2} \mathrm{~d} x^{\mu} \mathrm{d} x_{\mu} \\
& =\frac{1}{y^{2}}\left(\mathrm{~d} y^{2}+\mathrm{d} x^{\mu} \mathrm{d} x_{\mu}\right) \\
& =-\left(1+r^{2}\right) \mathrm{d} t^{2}+\frac{\mathrm{d} r^{2}}{1+r^{2}}+r^{2} \mathrm{~d} \Omega_{2}^{2} .
\end{aligned}
$$

or in global coordinates

$$
\mathrm{d} s_{A d S_{4}}^{2}=-\cosh ^{2} \rho \mathrm{d} t^{2}+\mathrm{d} \rho^{2}+\sinh ^{2} \rho \mathrm{d} \Omega_{2}^{2},
$$

where $\mathrm{d} \Omega_{2}^{2}=\mathrm{d} \vartheta^{2}+\sin ^{2} \vartheta \mathrm{d} \varphi^{2}$ with $\vartheta \in[0, \pi]$ and $\varphi \in[0,2 \pi]$, defines a two sphere $S^{2}$.

In addition, the metric of the complex projective space $\mathbb{C P}^{2}$, normalized in such a way that $R_{m n}^{\mathbb{C \mathbb { P } ^ { 2 }}}=6 g_{m n}^{\mathbb{C P}^{2}}$, see $[49,58]$, is

$$
\begin{aligned}
\mathrm{d} s_{\mathbb{C P}^{2}}^{2} & =\mathrm{d} \lambda^{2}+\frac{1}{4} \sin ^{2} \lambda\left\{\mathrm{d} \theta^{2}+\sin ^{2} \theta \mathrm{d} \phi^{2}+\cos ^{2} \lambda(\mathrm{d} \sigma+\cos \theta \mathrm{d} \phi)^{2}\right\} \\
& =\mathrm{d} \lambda^{2}+\frac{1}{4} \sin ^{2} \lambda\left\{\Sigma_{1}^{2}+\Sigma_{2}^{2}+\cos ^{2} \lambda \Sigma_{3}^{2}\right\}
\end{aligned}
$$

where $0 \leq 2 \lambda, \theta, \phi / 2, \sigma / 4 \leq \pi$. In this coordinate system, the Kähler potential can be written as

$$
\omega=\frac{\sin ^{2} \lambda}{2}(\mathrm{~d} \sigma+\cos \theta \mathrm{d} \phi)
$$

with Kähler-form $\mathcal{J}=\frac{1}{2} \mathrm{~d} \omega$ and volume form $\operatorname{vol}\left(\mathbb{C P}^{2}\right)=\frac{i^{2}}{2} \mathcal{J} \wedge \mathcal{J}$ and the volume of $\mathbb{C P}^{2}$ is $\int \operatorname{vol}\left(\mathbb{C P}^{2}\right)=\frac{\pi^{2}}{2}$. Since, for fixed $\sigma$ and $\lambda=\pi / 2$ we have a $\mathbb{C P}^{1}$ then

$$
\int_{\mathbb{C P}^{1}} \mathcal{J}=-\pi \quad \text { and } \quad \int_{\mathbb{C P}^{2}} \mathcal{J} \wedge \mathcal{J}=-\pi^{2}
$$

Finally, it will be useful to consider the SU(2) symmetry of this metric. Then, we can write the metric in terms of the Maurer-Cartan forms of the group SU(2) given by

$$
\begin{aligned}
& L_{1}=\frac{1}{\sqrt{2}}(-\sin \sigma d \theta+\cos \sigma \sin \theta d \phi) \equiv \frac{1}{\sqrt{2}} \Sigma_{1} \\
& L_{2}=\frac{1}{\sqrt{2}}(\cos \sigma d \theta+\sin \sigma \sin \theta d \phi) \equiv \frac{1}{\sqrt{2}} \Sigma_{2} \\
& L_{3}=\frac{1}{\sqrt{2}}(d \sigma+\cos \theta d \phi) \equiv \frac{1}{\sqrt{2}} \Sigma_{3} .
\end{aligned}
$$

that satisfies $\mathrm{d} L^{i}=\frac{\epsilon^{i j k}}{\sqrt{2}} L^{j} \wedge L^{k}$ and $\Sigma_{1} \wedge \Sigma_{2} \wedge \Sigma_{3}=\sin \theta \mathrm{d} \theta \wedge \mathrm{d} \sigma \wedge \mathrm{d} \phi$. 
Open Access. This article is distributed under the terms of the Creative Commons Attribution License (CC-BY 4.0), which permits any use, distribution and reproduction in any medium, provided the original author(s) and source are credited.

\section{References}

[1] J.M. Maldacena, The large- $N$ limit of superconformal field theories and supergravity, Int. J. Theor. Phys. 38 (1999) 1113 [hep-th/9711200] [INSPIRE].

[2] O. Aharony, O. Bergman, D.L. Jafferis and J. Maldacena, $N=6$ superconformal Chern-Simons-matter theories, M2-branes and their gravity duals, JHEP 10 (2008) 091 [arXiv:0806.1218] [INSPIRE].

[3] A. Guarino, D.L. Jafferis and O. Varela, String theory origin of dyonic $N=8$ supergravity and its Chern-Simons duals, Phys. Rev. Lett. 115 (2015) 091601 [arXiv:1504.08009] [INSPIRE].

[4] A. Guarino, J. Tarrio and O. Varela, Romans-mass-driven flows on the D2-brane, JHEP 08 (2016) 168 [arXiv: 1605.09254] [INSPIRE].

[5] J.H. Schwarz, Superconformal Chern-Simons theories, JHEP 11 (2004) 078 [hep-th/0411077] [INSPIRE].

[6] B. de Wit and H. Nicolai, $N=8$ supergravity, Nucl. Phys. B 208 (1982) 323 [InSPIRE].

[7] B. de Wit and H. Nicolai, The consistency of the $S^{7}$ truncation in $D=11$ supergravity, Nucl. Phys. B 281 (1987) 211 [inSPIRE].

[8] H. Nicolai and K. Pilch, Consistent truncation of $D=11$ supergravity on $A d S_{4} \times S^{7}$, JHEP 03 (2012) 099 [arXiv:1112.6131] [INSPIRE].

[9] G. Dall'Agata, G. Inverso and M. Trigiante, Evidence for a family of $\mathrm{SO}(8)$ gauged supergravity theories, Phys. Rev. Lett. 109 (2012) 201301 [arXiv:1209.0760] [InSPIRE].

[10] G. Dall'Agata, G. Inverso and A. Marrani, Symplectic deformations of gauged maximal supergravity, JHEP 07 (2014) 133 [arXiv:1405.2437] [INSPIRE].

[11] B. de Wit and H. Nicolai, Deformations of gauged $\mathrm{SO}(8)$ supergravity and supergravity in eleven dimensions, JHEP 05 (2013) 077 [arXiv:1302.6219] [INSPIRE].

[12] K. Lee, C. Strickland-Constable and D. Waldram, New gaugings and non-geometry, arXiv: 1506.03457 [INSPIRE].

[13] C.M. Hull, A new gauging of $N=8$ supergravity, Phys. Rev. D 30 (1984) 760.

[14] A. Guarino and O. Varela, Dyonic ISO(7) supergravity and the duality hierarchy, JHEP 02 (2016) 079 [arXiv: 1508.04432] [INSPIRE].

[15] C.M. Hull and N.P. Warner, Noncompact gaugings from higher dimensions, Class. Quant. Grav. 5 (1988) 1517 [INSPIRE].

[16] A. Guarino and O. Varela, Consistent $N=8$ truncation of massive IIA on $S^{6}$, JHEP 12 (2015) 020 [arXiv:1509.02526] [INSPIRE].

[17] O. Varela, $A d S_{4}$ solutions of massive IIA from dyonic $\mathrm{ISO}(7)$ supergravity, JHEP 03 (2016) 071 [arXiv:1509.07117] [InSPIRE].

[18] Y. Pang and J. Rong, $N=3$ solution in dyonic ISO(7) gauged maximal supergravity and its uplift to massive type IIA supergravity, Phys. Rev. D 92 (2015) 085037 [arXiv:1508.05376] [INSPIRE]. 
[19] Y. Pang and J. Rong, Evidence for the holographic dual of $N=3$ solution in massive type IIA, Phys. Rev. D 93 (2016) 065038 [arXiv:1511.08223] [InSPIRE].

[20] M. Fluder and J. Sparks, D2-brane Chern-Simons theories: F-maximization = a-maximization, JHEP 01 (2016) 048 [arXiv:1507.05817] [INSPIRE].

[21] M. Cvetič, H. Lü and C.N. Pope, Gauged six-dimensional supergravity from massive type IIA, Phys. Rev. Lett. 83 (1999) 5226 [hep-th/9906221] [INSPIRE].

[22] E. Bergshoeff, R. Kallosh, T. Ortín, D. Roest and A. Van Proeyen, New formulations of $D=10$ supersymmetry and D8-O8 domain walls, Class. Quant. Grav. 18 (2001) 3359 [hep-th/0103233] [INSPIRE].

[23] D. Marolf, Chern-Simons terms and the three notions of charge, in Quantization, gauge theory, and strings. Proceedings, International Conference dedicated to the memory of Professor Efim Fradkin, Vol. 1+2, Moscow Russia, 5-10 June 2000, pg. 312 [hep-th/0006117] [INSPIRE].

[24] O. Bergman and G. Lifschytz, Branes and massive IIA duals of 3d CFT's, JHEP 04 (2010) 114 [arXiv: 1001.0394] [INSPIRE].

[25] F. Apruzzi, M. Fazzi, D. Rosa and A. Tomasiello, All AdS $S_{7}$ solutions of type-II supergravity, JHEP 04 (2014) 064 [arXiv:1309.2949] [INSPIRE].

[26] F. Apruzzi, M. Fazzi, A. Passias and A. Tomasiello, Supersymmetric AdS $S_{5}$ solutions of massive IIA supergravity, JHEP 06 (2015) 195 [arXiv: 1502.06620] [INSPIRE].

[27] A. Rota and A. Tomasiello, AdS $S_{4}$ compactifications of $A d S_{7}$ solutions in type-II supergravity, JHEP 07 (2015) 076 [arXiv:1502.06622] [INSPIRE].

[28] O. Aharony, D. Jafferis, A. Tomasiello and A. Zaffaroni, Massive type IIA string theory cannot be strongly coupled, JHEP 11 (2010) 047 [arXiv: 1007.2451] [INSPIRE].

[29] M.B. Green, C.M. Hull and P.K. Townsend, D-brane Wess-Zumino actions, T-duality and the cosmological constant, Phys. Lett. B 382 (1996) 65 [hep-th/9604119] [INSPIRE].

[30] B. Janssen, Y. Lozano and D. Rodriguez-Gomez, The baryon vertex with magnetic flux, JHEP 11 (2006) 082 [hep-th/0606264] [INSPIRE].

[31] N. Gutierrez, Y. Lozano and D. Rodriguez-Gomez, Charged particle-like branes in ABJM, JHEP 09 (2010) 101 [arXiv: 1004.2826] [InSPIRE].

[32] Y. Lozano, M. Picos, K. Sfetsos and K. Siampos, ABJM baryon stability and Myers effect, JHEP 07 (2011) 032 [arXiv:1105.0939] [INSPIRE].

[33] D.S. Freed and E. Witten, Anomalies in string theory with D-branes, Asian J. Math. 3 (1999) 819 [hep-th/9907189] [INSPIRE].

[34] O. Aharony, A. Hashimoto, S. Hirano and P. Ouyang, D-brane charges in gravitational duals of $2+1$ dimensional gauge theories and duality cascades, JHEP 01 (2010) 072 [arXiv:0906.2390] [INSPIRE].

[35] D. Gaiotto and A. Tomasiello, The gauge dual of Romans mass, JHEP 01 (2010) 015 [arXiv: 0901.0969] [INSPIRE].

[36] E. Witten, Baryons and branes in anti-de Sitter space, JHEP 07 (1998) 006 [hep-th/9805112] [INSPIRE].

[37] P.-S. Hsin and N. Seiberg, Level/rank duality and Chern-Simons-matter theories, JHEP 09 (2016) 095 [arXiv: 1607.07457] [INSPIRE]. 
[38] S.G. Naculich, H.A. Riggs and H.J. Schnitzer, Group level duality in WZW models and Chern-Simons theory, Phys. Lett. B 246 (1990) 417 [InSPIRE].

[39] E.J. Mlawer, S.G. Naculich, H.A. Riggs and H.J. Schnitzer, Group level duality of WZW fusion coefficients and Chern-Simons link observables, Nucl. Phys. B 352 (1991) 863 [INSPIRE].

[40] S.G. Naculich and H.J. Schnitzer, Level-rank duality of the U(N) WZW model, Chern-Simons theory and 2D qYM theory, JHEP 06 (2007) 023 [hep-th/0703089] [INSPIRE].

[41] A. Brandhuber, N. Itzhaki, J. Sonnenschein and S. Yankielowicz, Baryons from supergravity, JHEP 07 (1998) 020 [hep-th/9806158] [INSPIRE].

[42] J.M. Maldacena, Wilson loops in large- $N$ field theories, Phys. Rev. Lett. 80 (1998) 4859 [hep-th/9803002] [INSPIRE].

[43] J. Sonnenschein, What does the string/gauge correspondence teach us about Wilson loops?, in Supersymmetry in the theories of fields, strings and branes. Proceedings, Advanced School, Santiago de Compostela Spain, 26-31 July 1999, pg. 219 [hep-th/0003032] [INSPIRE].

[44] J. McGreevy, L. Susskind and N. Toumbas, Invasion of the giant gravitons from anti-de Sitter space, JHEP 06 (2000) 008 [hep-th/0003075] [INSPIRE].

[45] M.T. Grisaru, R.C. Myers and O. Tafjord, SUSY and Goliath, JHEP 08 (2000) 040 [hep-th/0008015] [INSPIRE].

[46] A. Hashimoto, S. Hirano and N. Itzhaki, Large branes in AdS and their field theory dual, JHEP 08 (2000) 051 [hep-th/0008016] [INSPIRE].

[47] T. Nishioka and T. Takayanagi, Fuzzy ring from M2-brane giant torus, JHEP 10 (2008) 082 [arXiv:0808.2691] [INSPIRE].

[48] A. Hamilton, J. Murugan, A. Prinsloo and M. Strydom, A note on dual giant gravitons in $A d S_{4} \times C P^{3}, J H E P 04(2009) 132$ [arXiv: 0901.0009] [inSPIRE].

[49] C. Cardona and H. Nastase, Open strings on D-branes from ABJM, JHEP 06 (2015) 016 [arXiv: 1407.1764] [INSPIRE].

[50] M. Herrero, Y. Lozano and M. Picos, Dielectric 5-branes and giant gravitons in ABJM, JHEP 08 (2011) 132 [arXiv:1107.5475] [INSPIRE].

[51] C. Núñez, M. Piai and A. Rago, Wilson loops in string duals of walking and flavored systems, Phys. Rev. D 81 (2010) 086001 [arXiv:0909.0748] [INSPIRE].

[52] S.S. Gubser, I.R. Klebanov and A.M. Polyakov, A semiclassical limit of the gauge/string correspondence, Nucl. Phys. B 636 (2002) 99 [hep-th/0204051] [InSPIRE].

[53] S. Ryu and T. Takayanagi, Holographic derivation of entanglement entropy from AdS/CFT, Phys. Rev. Lett. 96 (2006) 181602 [hep-th/0603001] [INSPIRE].

[54] I.R. Klebanov, D. Kutasov and A. Murugan, Entanglement as a probe of confinement, Nucl. Phys. B 796 (2008) 274 [arXiv:0709.2140] [INSPIRE].

[55] D.M. Hofman and J.M. Maldacena, Giant magnons, J. Phys. A 39 (2006) 13095 [hep-th/0604135] [INSPIRE].

[56] D.E. Berenstein, J.M. Maldacena and H.S. Nastase, Strings in flat space and pp waves from $N=4$ super Yang-Mills, JHEP 04 (2002) 013 [hep-th/0202021] [INSPIRE].

[57] T. Araujo, G. Itsios, H. Nastase and E.Ó. Colgáin, Penrose limits and spin chains in the GJV/CS-SYM duality, arXiv: 1706.02711 [INSPIRE].

[58] C.N. Pope, Eigenfunctions and $\operatorname{Spin}(c)$ structures in $C P^{2}$, Phys. Lett. B 97 (1980) 417 [INSPIRE]. 